\title{
Predicting and reasoning about replicability using structured groups
}

Bonnie C. Wintle ${ }^{1}$, Fallon Mody ${ }^{2}$, Eden T. Smith ${ }^{2}$, Anca M. Hanea ${ }^{1}$, David P. Wilkinson ${ }^{1}$, Victoria Hemming $^{3}$, Martin Bush ${ }^{2}$, Hannah Fraser ${ }^{1}$, Felix Singleton-Thorn ${ }^{4}$, Marissa F. McBride ${ }^{1,5}$, Elliot Gould $^{1}$, Andrew Head ${ }^{2}$, Dan Hamilton ${ }^{2}$, Libby Rumpff ${ }^{1}$, Rink Hoekstra ${ }^{6}$, Fiona Fidler ${ }^{1,2}$

\footnotetext{
${ }^{1}$ MetaMelb, School of BioSciences, The University of Melbourne, Victoria, Australia

${ }^{2}$ MetaMelb, School of Historical and Philosophical Studies, The University of Melbourne, Victoria, Australia

${ }^{3}$ Martin Conservation Decisions Lab, Department of Forest and Conservation Sciences, University of British Columbia, Vancouver, Canada

${ }^{4}$ School of Psychological Sciences, The University of Melbourne, Victoria, Australia

${ }^{5}$ Centre for Environmental Policy, Imperial College London, U.K.

${ }^{6}$ Department of Pedagogical and Educational Sciences, University of Groningen, The Netherlands
}

\begin{abstract}
This paper explores judgements about the replicability of social and behavioural sciences research, and what drives those judgements. Using a mixed methods approach, it draws on qualitative and quantitative data elicited using a structured iterative approach for eliciting judgements from groups, called the IDEA protocol ('Investigate', 'Discuss', 'Estimate' and 'Aggregate'). Five groups of five people separately assessed the replicability of 25 'known-outcome' claims. That is, social and behavioural science claims that have already been subject to at least one replication study. Specifically, participants assessed the probability that each of the 25 research claims will replicate (i.e. a replication study would find a statistically significant result in the same direction as the original study). In addition to their quantitative judgements, participants also outlined the reasoning behind their judgements. To start, we quantitatively analysed some possible correlates of predictive accuracy, such as self-rated understanding and expertise in assessing each claim, and updating of judgements after feedback and discussion. Then we qualitatively analysed the reasoning data (i.e., the comments and justifications people provided for their judgements) to explore the cues and heuristics used, and features of group discussion that accompanied more and less accurate judgements.
\end{abstract}

\section{Introduction}

Over the last decade, several large-scale projects have attempted to replicate the findings of original published research in preclinical medicine, economics and psychology. The results of these studies have arguably brought the evidence base in those disciplines into question (Begley \& Ellis 2012; Errington et al. 2014; Klein et al. 2014; Open Science Collaboration 2015; Klein et al. 2018). This 'replication crisis' seen in many areas of science is a significant problem. Not only because it undermines the evidence base for decision making, but also because it undermines trust in science. Unfortunately, the extent of the 'replication crisis' has not yet been evaluated. Outside of large-scale replication studies such as those cited above, attempts to directly replicate original research appear only rarely in the published literature (Kelly 2006; Schmidt 2009). The rarity of replication studies is uncontroversially explained by inappropriate publishing incentives 
(Hamilton et al. 2020) and funding incentives, offering researchers little motivation for engaging in such work (Nosek \& Bar-Anan 2012). Replication studies also face other logistical challenges, including obtaining methods, materials and data from the original researchers, and the time and resources required to conduct them (Nosek \& Errington 2020).

Given the challenges to conducting entire replication studies, judgements from people about the replicability of research may offer a cost-effective alternative for evaluating the reliability of the evidence base. Those that are considered unlikely to be replicable could then be the target of actual replication studies (Isager et al. 2020). Fortunately, previous research has shown that, in the aggregate, people are quite good at predicting research replicability in the social and behavioural sciences. Dreber et al. (2015) found the best results from a prediction market, with the market correctly classifying $71 \%$ of studies from the Reproducibility Project Psychology as being replicable (or not). This compared with 58\% for the simple survey. In social science, predictions about the replicability of 21 experiments published in Nature and Science achieved 86\% classification accuracy, i.e. aggregated estimates were on the 'right' side of 50\% in 18 of 21 studies, for both the prediction market and the simple survey (Camerer et al. 2018). And again, in psychology (Many Labs 2), classification accuracy was 75\% using the prediction market and $67 \%$ using the simple survey (Forsell et al. 2019). Simple surveys and prediction markets provide similar estimates, but survey predictions tend to be less extreme and therefore perform less well, when predictions are reasonably good to begin with.

While people seem to accurately judge the replicability of research in psychology and social sciences, results from other disciplines are more mixed. Forecasts of the replicability of five prominent studies in preclinical cancer research (Benjamin et al. 2017), based on replication studies conducted by Errington et al. (2014), indicated that it may be more difficult to predict replicability of cancer biology studies than of studies in the social and behavioural sciences. Forecasts in this study were over optimistic, with participants tending to believe that replication studies would achieve a statistically significant effect in the same direction as the original study. For experts, $73 \%$ of their 'significance' forecasts were above $50 \%$, when none of the five replication studies successfully replicated under the 'significance' criterion. In experimental economics, all predictions were greater than $50 \%$, so the classification accuracy equalled the replication rate of $61 \%$ for both the prediction market and the simple survey (Camerer et al. 2016).

How much does expertise matter in evaluating replicability? In Benjamin et al. (2017), experts and novices were similarly inaccurate at predicting replicability of preclinical cancer studies. Experts were more overconfident when they were more familiar with a study, and generally did not provide better forecasts than trainees in the study. On the other hand, the authors' observations "hint at the prospect" that researchers with higher h-index (a controversial measure of 'research impact') may be more accurate forecasters of replicability. To further investigate the question, a recent study (Hoogeveen et al. 2020) asked 233 people without a $\mathrm{PhD}$ ('laypeople') to predict replicability of 27 high-profile social-science findings (a subset of studies from Camerer et al 2018 and Forsell et al. 2018). Non expert predictions were above chance accuracy, at 59\%. (Accuracy increased to $67 \%$ when participants were additionally provided with a summarised description of the original study and calculated Bayes Factors). More detailed accounts of the role of expertise in replicability prediction are forthcoming (Marcoci et al., in prep). 
Unfortunately, it is difficult to know in advance who will be an accurate judge or forecaster, at least using common markers of 'expertise', such as publication record and expert status (Burgman et al. 2011b; Burgman 2015). Whilst we do reopen the question of potential relationships between accuracy and common markers of expertise in this paper, one of our goals is to explore whether we can better recognise the more accurate predictions using characteristics of reasoning and judgements, rather than characteristics of the experts themselves. One characteristic that may point to more or less accurate judgements is confidence. Whilst evidence suggests that more confident individuals are no more accurate on average (Burgman et al. 2011b), there is some evidence that judgement-level confidence, measured in various ways, contains potentially useful information. Koriat (2012) developed a heuristic for choosing between judgments of group members, called Maximum Confidence Slating (MCS). When faced with judgments from two or more people, the MCS heuristic selects the judgment of the most confident judge for that particular question (confidence in each response is also specified by the judge). Having flexibility to adopt judgments from different judges dispenses with the need to determine a priori who is the best judge. Using both perceptual stimuli (e.g. which of these lines is longer) and a general-knowledge task (e.g. which of two European countries has a larger area or population), results were favourable for MCS. It produced inferential accuracy higher than would be attained from choosing the best judge. For example, in Study 1, the percentage of correct decisions was 67.82 for the best judge, $66.98 \%$ for the lesser performing judge, and $69.88 \%$ when taking the judgment with the highest confidence attached. On adding a third participant to the pair, performance of MCS heuristic improved even more $(71.88 \%)[\mathrm{t}(18)=5.05, \mathrm{p}<0.0001]$.

Taking an aggregated judgement from multiple experts is almost always preferable to relying on a single expert. The wisdom of crowds phenomenon shows us that individual biases tend to cancel each other out in the aggregate by bracketing the true value (Larrick \& Soll 2006). We can further improve on the wisdom of crowds by a) eliciting judgements in a structured way (e.g., Soll \& Klayman 2004) and b) by allowing diverse group members to share information and interrogate each others' judgements. Although some researchers argue that group interaction and social influence can undermine the wisdom of crowds effect by diminishing diversity (Lorenz et al. 2011), leading to group think (Janis 1982), and precipitating more overconfidence in the accuracy of their collective judgment (Sniezek \& Henry 1990), others believe that the benefits of information sharing are clear (e.g., Burgman et al. 2011a; Farrell 2011; Hemming et al. 2018b). Discussion offers the potential to improve group performance. It can resolve misunderstanding of the question and provide an opportunity for people to introduce new information and learn from each other. Discussions may improve group performance by drawing out hidden information (Stasser \& Titus 2003; Mojzisch \& Schulz-Hardt 2010), encouraging critical thinking (Postmes et al. 2001) and counter-factual reasoning (Galinsky \& Kray 2003), and displaying and resolving differential motivational contingencies (Önkal et al. 2011). Group interaction also allows people to communicate their degree of confidence to each other, which can also bolster judgement performance (Bahrami et al. 2010).

We argue that, although contingent on many factors, interaction is beneficial to group performance. Moreover, carefully structuring the way we elicit and aggregate predictions from groups, and managing group interactions, may further improve the accuracy and calibration of judgements. One such structured elicitation approach is the 'IDEA protocol' ('Investigate', 'Discuss', 'Estimate' and 'Aggregate') (Hanea et al. 2017; Hemming et al. 2018a), an iterative 
Delphi-style method that integrates techniques for mitigating some of the biases of human judgement (particularly overconfidence) and draws on the collective wisdom of groups, in a transparent and interactive way (see Methods section for more detail). It also combines the judgements of individuals using mathematical (French 2011) rather than behavioural aggregation approaches, so group members are not forced to agree on a consensus judgement. IDEA is particularly advantageous when experts have private information that would be valuable to a group judgement, as is likely the case in this context, given the role of private insights in previous replicability predictions (Dreber et al. 2015). In Intelligence, structured 'IDEA groups' (Hanea et al. 2017) have provided more accurate geopolitical forecasts than a benchmark commissioned by the US Government (linear pool of forecasts), and performed similarly to prediction markets (Burgman 2015). In the same geopolitical forecasting tournament, results from the Good Judgment Project team showed that trained teams of 'superforecasters' outperformed all other individuals, crowds, and other research teams (Mellers et al. 2014; Mellers et al. 2015a; Mellers et al. 2015b), and when previously-validated aggregation techniques were applied, self-reported forecasts ('beliefs') of geopolitical events were at least as accurate as prediction-market prices (Dana et al. 2019). Similar benefits of structured group elicitations and considered aggregation methods on improving judgement accuracy can be seen in environmental science (McBride et al. 2012; Wintle et al. 2013; Hemming et al. 2018b) and engineering (Hemming et al. 2020).

In this paper, we are taking the structured group elicitation protocols developed and tested in other domains (intelligence, environmental science, engineering) and applying them to the elicitation of replicability predictions in social and behavioural science claims. Exploring group deliberation and reasoning about what makes a result reliable (or not) may provide further insights into quantitative judgements about replicability (what drives their judgements), and when people update their judgements in light of discussion. Our paper explores markers of expertise that are often deployed to select experts, some other judgement characteristics that indicate claim-level confidence, as well as the rich qualitative reasoning data that arises when assessing the reliability of research claims.

\section{Hypotheses and Exploratory questions}

The main purpose of this paper is to better understand how people make judgements about the reliability of research, and how they reason around these judgements. In doing so, the paper examines some correlates of predictive accuracy, particularly, which, if any, of our demographics, confidence and expertise measures correlate with the ability to predict the outcome of replication studies. Aside from looking at relationships with accuracy, this paper also explores other characteristics of judgements and judges, such as the relationship between confidence and expertise, and participant willingness to change their mind (judgements) after discussion with the group. We also explore the qualitative reasoning used by individual participants to support their quantitative judgements, and to provide context for our quantitative results. This includes examples of cues people use when they change their mind (shift judgements the most after discussion), when they are relatively (in)accurate, and when they consider themselves to be (non) expert in that field / claim.

The key quantitative hypotheses and analyses that underpin this paper are preregistered on the Open Science Framework (https://osf.io/e8dh7/). Below, we outline these, together with other 
research questions that we explore here. The four preregistered confirmatory hypotheses predict an effect in a particular direction. Exploratory research questions are not accompanied by such clear directional statements unless indicated.

Table 1. Hypotheses and research questions

\begin{tabular}{|c|c|c|c|}
\hline Topic & Level & Preregistered & Research Question / Hypothesis \\
\hline Accuracy & Participant & Confirmatory & $\begin{array}{l}\text { Hypothesis } 1: \text { Round } 2 \text { judgements (post- } \\
\text { discussion) will be more accurate than Round } 1 \\
\text { judgements (pre-discussion) }\end{array}$ \\
\hline Accuracy & Participant & Confirmatory & $\begin{array}{l}\text { Hypothesis 2: Those who perform well on a quiz } \\
\text { testing familiarity with previous research and } \\
\text { concepts related to assessments of replicability } \\
\text { will be more accurate on the main task. }\end{array}$ \\
\hline Accuracy & Participant & Exploratory & $\begin{array}{l}\text { Question 1a: What are the demographic, } \\
\text { experience and psychometric characteristics of } \\
\text { more accurate judges? }\end{array}$ \\
\hline Updating & Participant & Exploratory & $\begin{array}{l}\text { Question 1b: What are the demographic, } \\
\text { experience and psychometric characteristics of } \\
\text { those who update their judgements most between } \\
\text { rounds? }\end{array}$ \\
\hline $\begin{array}{l}\text { Updating / } \\
\text { Accuracy }\end{array}$ & Participant & Exploratory & $\begin{array}{l}\text { Question 2: Are those who update their } \\
\text { judgements between rounds more accurate than } \\
\text { other participants in Round } 2 \text { (after discussion, } \\
\text { across all claims)? }\end{array}$ \\
\hline Updating & Participant & Confirmatory & $\begin{array}{l}\text { Hypothesis 3: Those who update (shift) their } \\
\text { estimates most between rounds will be less } \\
\text { confident in their judgements on average, as } \\
\text { indicated by: (i) lower mean claim-level expertise } \\
\text { ratings, (ii) lower mean claim-level understanding } \\
\text { ratings. }\end{array}$ \\
\hline $\begin{array}{l}\text { Accuracy } \\
\text { Confidence }\end{array}$ & Judgement & Confirmatory & $\begin{array}{l}\text { Hypothesis 4: Judgements will be more accurate } \\
\text { when associated with signals of higher confidence } \\
\text { on those particular judgements, specifically, (i) } \\
\text { higher claim expertise ratings, (ii) higher claim } \\
\text { understanding ratings, (iii) more certain } \\
\text { (narrower) intervals. }\end{array}$ \\
\hline $\begin{array}{l}\text { Improvement / } \\
\text { Confidence }\end{array}$ & Participant & Exploratory & $\begin{array}{l}\text { Question 3: Do those with lower confidence } \\
\text { across claims (signalled by (i) lower average } \\
\text { claim expertise ratings, (ii) lower average claim } \\
\text { understanding ratings) improve more after } \\
\text { discussion? (become more accurate) }\end{array}$ \\
\hline
\end{tabular}




\section{Method}

The preregistration is available on the Open Science Framework project page (https://osf.io/8y2ra/). All materials and anonymised data, including R code used for analyses will be made available on completion of the project at the same link. Although data had been collected prior to preregistration, the author of the preregistration was blinded to the data and any preliminary analyses before uploading the preregistration, and was not directly involved in data collection. This study was approved as part of the larger repliCATS project (Collaborative Assessment of Trustworthy Science) by the ethics board at the University of Melbourne (Ethics ID: 1853445).

The Plain Language Statement and demographics form are available on the Open Science Framework (OSF) page for this project. As we are still recruiting participants for the larger SCORE (Systematizing Confidence in Open Research and Evidence) project, we have not yet made the quiz and claim materials publicly available but will do so at the end of the project.

\section{Participants}

The study was undertaken as part of a larger two-day workshop to assess the replicability of claims for the SCORE program. The main aim of the workshop was to obtain confidence scores related to the replicability of social and behavioural science claims, from groups, using the IDEA protocol. The workshop was scheduled alongside the Society for the Improvement of Psychological Science conference 2019 in Rotterdam, Netherlands. We offered participants bursaries (AUD400 if from the UK / EU and AUD1000 if outside of the UK / EU) to cover their travel expenses, allowing them to attend our workshop as well as the conference. Our resulting study sample consisted of those who applied for the bursaries: mainly academics, postgraduate and undergraduate students with an interest in open science and replicability. The majority of participants were from psychology and related disciplines, but this was not a requirement for participation.

In total, 156 participants were recruited to attend the larger workshop, of which 25 were randomly assigned to participate in this study.

\section{Sampling Plan}

A subset of groups at our larger workshop (5 of 30 groups) were randomly assigned to participate in this study. The five groups consisted of five individuals, and each was facilitated by a member of our research team. So, in total, our study involved $\mathrm{N}=25$ participants in five IDEA groups (5 groups x 5 participants), each answering 25 questions (i.e. assessing 25 'known-outcome' claims, i.e., social and behavioural science claims that have already been subject to at least one replication study).

Our sample size was determined by considering both logistics and the reliability of Brier scores. We did not run power analyses for this study, since pragmatic considerations of the larger project substantially influenced our sampling plan. Logistics: The number of participants we could assign to assessing 'known-outcome' claims (i.e. this study) depended on the number of 'unknown- 
outcome' claims (i.e. the main part of the SCORE project) we received to assess for the same 2day workshop. As we received 557 'unknown-outcome' claims to assess by 156 participants, this allowed us to dedicate 2 days x 25 participants' time to this study. Reliability of Brier scores: Brier scores measure the long run accuracy of participant forecasts. Previous analyses conducted by members of our team indicates that Brier scores are unstable if less than 20 binary outcome questions are answered by each participant. Other research teams involved in predicting the outcomes of geopolitical events (Mellers et al. 2015b) have also previously specified a minimum of 25 questions for reliability. We settled on a design that required each participant to answer 25 binary outcome questions (i.e. to assess replicability of 25 claims). This was also to account for possible variation in the difficulty of claims. We stopped collecting data for this project at the end of our 2-day workshop (stopping rule determined by pre-allocated workshop time).

Prior to attending the workshop, participants completed some demographics questions that were used to allocate participants into groups, aiming for balance with respect to gender, region (European / non-European resident) and education (students vs non-students) (see supplementary material for further information).

Groups were facilitated by members of our research team who were blinded to the claims for assessment (i.e. they did not know the outcomes themselves). We were concerned that participants might recognize some of the claims and either share the 'answer' or unduly influence others in discussion, so facilitators set some ground rules at the start of the workshop, asking participants to abstain from discussion if they were confident that they ' $\mathrm{knew}$ ' the outcome. Facilitators were all briefed beforehand and provided a procedural checklist of items to cover and explain to participants, to promote consistency between groups. Specifically, they were given instructions and suggestions for prompting discussion, considering counterfactuals, encouraging a range of opinions to be expressed, together with guidelines around time to spend on each claim, tips for answering and interpreting each question on the platform, and training materials, e.g. to help participants interpret common statistics they may see reported in the papers they were evaluating.

\section{Materials}

We selected 25 claims to be evaluated from a database of 341 claims compiled from the major replication projects to date from the social and behavioural sciences (provided by the Center for Open Science). Using this database of known-outcome claims, we first applied some exclusion criteria. Specifically, the claims were reduced down from the original set using the following rules:

(i) Selected claims were from Many Labs 1 (Klein et al. 2014), Many Labs 2 (Klein et al. 2018), and Many Labs 3 (Ebersole et al. 2016), the Social Sciences Replication Project (Nature and Science) (Camerer et al. 2018) or the original Reproducibility Project Psychology (RPP) (Open Science Collaboration 2015).

(ii) The replication had at least $90 \%$ power to detect an effect $75 \%$ of size of that seen in the original study (calculated using fisher- $Z$ transformed correlation coefficients) 
(iii) Selected claims were not too famous (participants may already know the outcome of the replication study). See Supplementary material for the 10 studies we excluded for being too wellknown.

Prior to attending the workshop, participants completed their consent and demographics forms, together with a short quiz in Qualtrics, testing their knowledge and understanding of statistical concepts, meta-research and other items relevant to the main task. They were also asked to access our training materials (including a glossary, videos and links to short e-courses; https://osf.io/a89nc/) to help familiarise themselves with relevant meta-research and statistical concepts relevant to assessing claims.

To evaluate claims, participants answered a set of questions on an online repliCATS platform we developed for the SCORE program (Pearson et al. 2021). These are shown in Figure 1.

\section{Procedure}

Prior to data collection, anonymised usernames, groupings and claims were imported into our online repliCATS platform. Groups were assigned a facilitator (a member of the repliCATS team). In a face-to-face setting, but using the online platform, each of the five groups evaluated the same 25 'known-outcome' claims. That is, they evaluated empirically-tested claims made in research papers from the social and behavioural science literature that have already been subject to at least one replication study (see 'Materials' for more details of how claims were selected). A summary of the procedure is provided below, but the further details, including question wording, can be found in the paper introducing the repliCATS approach (Fraser et al. 2021).

To evaluate claims, groups were stepped through the IDEA protocol (Hanea et al. 2017) for capturing expert predictions about replicability of research claims (Figure 2). After logging on to the platform, participants saw a claim summary that included key statistics, such as details of the inferential test used in the paper, the effect measure, effect size, and sample size. After reading the research claim summary, participants investigated the paper further, i.e., they were provided a link to the full paper. Next, they entered individual Round 1 responses into the platform.

In the main quantitative task for Round 1, participants were asked to assess the probability that these claims would successfully replicate (i.e. a replication study would find a statistically significant result in the same direction as the original study). They were also asked to assess how well they understood the claim (1-7), whether they considered the underlying effect or relationship to be plausible (binary), and to rate their expertise in the field that the claim pertained to (off platform, 1-7). 


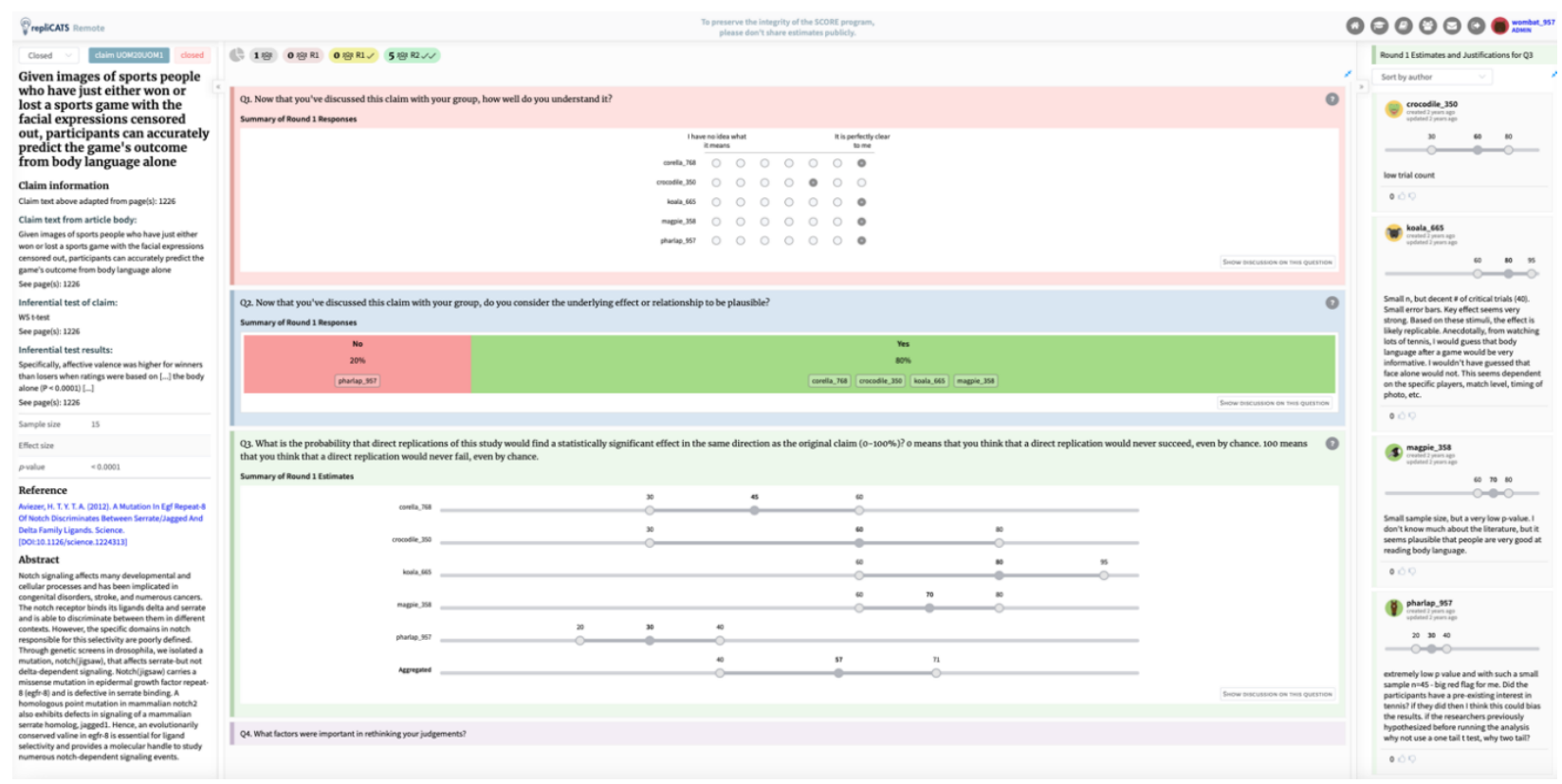

Figure 1. The online repliCATS platform that participants used to evaluate research claims.

Replicability estimates (question 3 on the platform) were then entered using a structured format for eliciting interval judgements (Burgman 2015); that is, participants provided their (i) lower bound, (ii) upper bound and (iii) best estimate of the probability that the claim would successfully replicate. This interval format aims to reduce overconfidence by breaking the question down into multiple steps (Soll \& Klayman 2004; Teigen \& Jørgensen 2005; Speirs-Bridge et al. 2010). When providing estimates of their bounds, participants are explicitly asked to consider reasons for and against their judgements (Koriat et al. 1980), i.e. think of reasons why a claim may successfully replicate when providing upper bounds on the probability, and then why it may not successfully replicate when providing lower bounds. Participants were also asked to enter the reasoning behind their judgements into the platform. For example, they might justify a low probability judgement by commenting on the small sample size of the original study, or how the constructs under investigation were operationalised. Participants had on average $\sim 15$ minutes to read through the claim and provide their private Round 1 judgements.

Following submission of the Round 1 judgements group members' anonymous estimates and reasoning were revealed during feedback, and the group could compare and discuss their estimates, share information and cross-examine reasoning and evidence. After discussing / hearing any new insights gained through discussion, group members individually entered a second and final private estimate. Round 2 estimates were combined using mathematical aggregation (French 2011), which, in this case, was a simple arithmetic mean. 


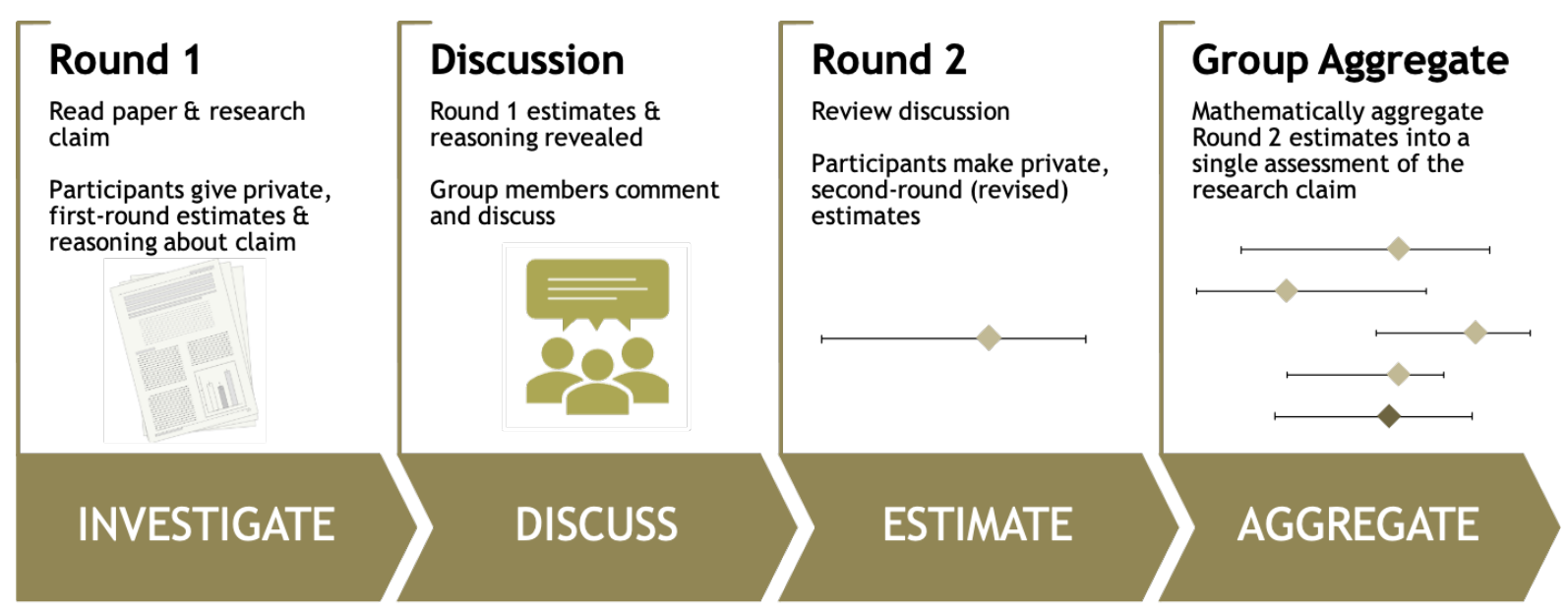

Figure 2. Overview of the IDEA protocol, as adopted in the repliCATS project

There was some flexibility around the order and manner in which claims were assessed between groups, e.g., some groups preferred to complete Round 1 in batches of 3-5 claims before moving on to the Discussion / Round 2 phase, whereas others worked through the full IDEA process, claim by claim. At the start of the workshop, claims were listed on the platform in the same order for all groups. We did not set rules about working through the claims in any particular order. Throughout the process, the ordering of claims on the repliCATS platform changes, as it is sorted by which round they are in, with claims still in Round 1 followed by claims in Round 2. This helps individuals track the progress of claims they have completed (Pearson et al. 2021).

\section{Measures}

\section{Accuracy}

We calculated the accuracy of participants' best estimates (Round 1 and Round 2 probabilities) using a distance (error) measure from the 'true value'. In this case, the 'true value' is a binary outcome for whether or not the claim replicated $(0,1)$.

For accuracy measures at the judgement level, we used a simple distance measure, i.e., the absolute error between the estimated probability of replication (converted from percentage to proportion) and 0 or 1 .

For participant-level and claim-level accuracy, the distance measure was combined into Brier scores (Brier 1950) to measure the long-run accuracy of individual participants over different claims, and the accuracy of different participants on individual claims, using the following:

$$
B S=\frac{1}{N} \sum_{c=1}^{N}\left(f_{c}-o_{c}\right)^{2}
$$


In which $f_{c}$ is a participant's prediction of replicability, $o_{c}$ the actual outcome of the replication attempt at claim $c$ ( $o_{c}$ is either 0 or 1: did the claim successfully replicate or not), and $N$ is the number of predictions the participant made.

\section{Precision}

For this measure, we calculated an interval width between 0 and 1 for each interval judgement by subtracting the lower bound from the upper bound.

\section{Updating (Shifting)}

We hypothesized that some variables will be associated with participants' propensity to change their mind after discussion and seeing other people's estimates. We measured the shift in estimates between rounds as Round 2 minus Round 1, separately for best estimates and interval widths.

\section{Prior knowledge}

At the participant level, for both initial and final estimates (i.e. Round 1 and Round 2 estimates, analyzed separately), we hypothesised that predictive accuracy may be positively associated with performance on a quiz testing familiarity with previous research and concepts related to assessments of replicability. Quiz scores ranged from 0-22 (when scored one point per each of the 22 questions). Quiz questions will be made public at the end of data collection for the broader project.

\section{Expertise}

We also hypothesised that, at the participant level, changing estimates between rounds may be negatively associated with overall claim expertise (self-reported, ordinal variable scored 1-7, averaged for the participant) and overall claim understanding (self-reported, ordinal variable scored 1-7, averaged for the participant). We acknowledge the long-debated concerns with averaging ordinal scales (assuming equidistant intervals from 1-7) to gain a single score per participant, and so interpret those particular measures with caution.

At the judgement level, for both initial and final estimates (i.e., Round 1 and Round 2, analyzed separately), we hypothesised that predictive accuracy may be positively associated with claimlevel expertise, claim-level understanding and interval width (narrower intervals, standardized interval width measure).

\section{Analysis}

Due to the groupings in the study design, we expected a cluster effect in Round 2 judgements, so we opted for modelling that accounts for this. We fitted linear mixed models in R (R Core Team 2020) using the nlme (Pinheiro et al. 2020), lme4 (Bates et al. 2015) and lmerTest (Kuznetsova et al. 2017) packages, and accounted for the variation arising from different groups as well as from repeated measures ( 25 questions per participant), by including these variables as random effects. 
In addition to our main preregistered analyses, we also conduct some additional analyses as flagged below. The order of analysis presented here follows the "topic" ordering of Table 1 in our Hypotheses and Exploratory Questions section above, and is again repeated in our presentation of Results and Discussion section below. The description of our qualitative analysis is at the end of this section, but in our Results and Discussion, quantitative and qualitative results appear together, with qualitative examples appearing with what we identify as the most relevant accompanying hypothesis.

\section{Quantitative Analysis}

We calculate $95 \%$ confidence intervals (CIs) and use 'inference by eye' comparisons (Cumming 2012) to infer differences between groups. That is, differences will be deemed statistically significant at the equivalent of alpha $<0.05$ when there is less than $25 \%$ overlap between groups' $95 \%$ confidence intervals. We also present results ( $p$ values and CIs) of a t test.

For hypotheses $2-4$, we calculated $p$ values and plot standardized model coefficients with 95\% CIs. Our inferences and conclusions consider both pieces of information.

No data was excluded from confirmatory analyses. Missing data points were treated as NAs, participants with NA data were included in the analyses.

\section{Hypothesis 1: Round 2 judgements (post-discussion) will be more accurate than Round 1 judgements (pre-discussion) (at the participant level)}

We investigate improvements in accuracy in final judgements by plotting participants' Average Brier scores and 95\% CIs before (Round 1) and after discussion (Round 2).

Additional exploratory analysis: To further test this hypothesis, we also perform a t-test for difference between groups of paired data, treating pre-/post-discussion as pre-/post-test.

Hypothesis 2: Those who perform well on a quiz testing familiarity with previous research and concepts related to assessments of replicability will be more accurate on the main task (at the participant level).

We separately modelled individuals' accuracy in Round 1 and Round 2 (Brier Scores) as a function of their quiz scores (Equations 2 and 3, respectively). We included a random effect for group in both Round 1 and Round 2 analyses. Even though the group effect would presumably be less for Round 1 than Round 2, there is still potential for facilitator influence (e.g. explaining instructions, allowing different procedures for assessing claims), different settings (e.g. some groups shared large rooms, which may have been noisier) and other group level effects (e.g. a particularly pessimistic or optimistic group might affect Round 1 judgements made later in the workshop). We note that the preregistration incorrectly included 'claim' as a random effect, which is not possible since participant-level accuracy is already aggregated across claims. 


$$
\begin{gathered}
\text { Round_1_Brier_Score } e_{i} \sim N\left(\alpha_{j[i]}+\beta_{1}(\text { quiz_score }), \sigma^{2}\right) \\
\alpha_{j} \sim N\left(\mu_{\alpha_{j}}, \sigma_{\alpha_{j}}^{2}\right) \text { for group } j=1, \ldots, J \\
\text { Round_2_Brier_Score } e_{i} \sim N\left(\alpha_{j[i]}+\beta_{1}(\text { quiz_score }), \sigma^{2}\right) \\
\alpha_{j} \sim N\left(\mu_{\alpha_{j}}, \sigma_{\alpha_{j}}^{2}\right) \text { for group } j=1, \ldots, J
\end{gathered}
$$

Additional exploratory analysis: We separately explore if scores on each of the two core components of the quiz (knowledge of statistical concepts and knowledge of meta-research) have the same relationship with accuracy, using Spearman rank correlations and 95\% CIs.

Exploratory Question 1: What are the demographic, experience and psychometric characteristics of (a) more accurate judges, and (b) those who update their judgements most between rounds?

In addition to the preregistered modelling, we also analysed additional information we collected from participants about their demographics and background. We collected information on gender, age, education, career stage, technical expertise (i.e. mathematics (generally), quantitative modelling / simulation, statistics, probability, experimental design, risk analysis, forecasting, 1-7), other relevant experience (with direct and/or partial / conceptual replication; forecasting replication studies; meta-research and/or preregistration, all binary), publications and training (background questions, https://osf.io/3hbs5/).

In the same survey, we also included ten items from the Actively Open-minded Thinking test (Stanovich \& West 1997), three items from the Cognitive Reflection Test (Frederick 2005), and four from the Berlin Numeracy test (Cokely et al. 2012) (https://osf.io/yehsn/). Two participants were excluded from these analyses due to not answering those questions.

We explore these relationships using Spearman Rank correlations and 95\% CIs for continuous / ordinal data $\mathrm{v}$ continuous data, and present interval plots and $95 \%$ CIs for continuous $\mathrm{v}$ categorical data. Precise statistical significance is also indicated with p-values.

\section{Exploratory Question 2: Are those who update their judgements between rounds more accurate than other participants in Round 2 (after discussion, across all claims)?}

We explore this question using separate linear mixed models, modelling participants' accuracy (individual Brier Scores across all claims) for each Round as a function of how much the participant shifted their judgment after discussion (Equations 4 and 5).

Shifting $_{\text {Best }}$ is defined as | Round 1 best estimate - Round 2 best estimate |. As for all previous models, we include a random effect for group. 


$$
\begin{gathered}
\text { Round_1_Brier_Score }_{i} \sim N\left(\alpha_{j[i]}+\beta_{1}\left(\text { Shifting }_{\text {Best }}\right), \sigma^{2}\right) \\
\alpha_{j} \sim N\left(\mu_{\alpha_{j}}, \sigma_{\alpha_{j}}^{2}\right) \text { for group } j=1, \ldots, J \\
\text { Round_2_Brier_Score }{ }_{i} \sim N\left(\alpha_{j[i]}+\beta_{1}(\text { Shifting } \text { Best }), \sigma^{2}\right) \\
\alpha_{j} \sim N\left(\mu_{\alpha_{j}}, \sigma_{\alpha_{j}}^{2}\right) \text { for group } j=1, \ldots, J
\end{gathered}
$$

\section{Hypothesis 3: Those who update (shift) their estimates most between rounds will be less confident in their judgements on average, as indicated by: (i) lower mean claim-level expertise ratings, (ii) lower mean claim-level understanding ratings (at the participant level).}

We modelled the difference in participants' best estimates (Equation 6), and the difference in their interval widths (Equation 7), between Round 1 and Round 2 as a function of mean expertise and mean understanding averaged across all claims for a given participant.

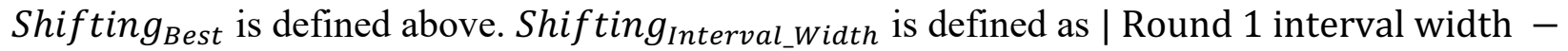
Round 2 interval width |. We include a random effect term for group to account for groupspecific variation.

$$
\begin{gathered}
\text { Shifting } \left._{\text {Best }_{i}} \sim N\left(\alpha_{j[i]}+\beta_{1} \text { (mean_expertise }\right)+\beta_{2}(\text { mean_understanding }), \sigma^{2}\right) \\
\alpha_{j} \sim N\left(\mu_{\alpha_{j}}, \sigma_{\alpha_{j}}^{2}\right) \text { for group } j=1, \ldots, J
\end{gathered}
$$

$$
\begin{gathered}
\text { Shifting } \left._{\text {Interval_Width }} \sim N\left(\alpha_{j[i]}+\beta_{1} \text { (mean_expertise }\right)+\beta_{2}(\text { mean_understanding }), \sigma^{2}\right) \\
\alpha_{j} \sim N\left(\mu_{\alpha_{j}}, \sigma_{\alpha_{j}}^{2}\right) \text { for group } j=1, \ldots, J
\end{gathered}
$$

NB: Since 'understanding' was elicited in both rounds, we needed to choose between Round 1 or Round 2 scores for this shifting analysis (not specified in the preregistration). Although it does not substantively affect results either way, we opted for Round 1 'understanding' scores here, since coming from a place of low understanding at the start might suggest that participants are more likely to shift. 
Hypothesis 4: Judgements will be more accurate when associated with signals of higher confidence on those particular judgements, specifically, (i) higher claim expertise ratings, (ii) higher claim understanding ratings, (iii) more certain (narrower) intervals.

We modelled the difference between individuals' best-estimates and the known replication outcome separately for each round (Equations 8 and 9). The difference for each round was modelled as a function of individuals' claim-level expertise, their understanding of the claim for that round, as well as their interval width for that round. In both models, we included random effects for participant, group and claim.

$$
\begin{gathered}
\text { Round_1_absolute_error }{ }_{i} \sim N\left(\mu, \sigma^{2}\right) \\
\mu=\alpha_{j[i], k[i], l[i]}+\beta_{1}(\text { claim_level_expertise })+\beta_{2}(\text { Round_1_claim_level_understanding }) \\
+\beta_{3}(\text { Round_1_claim_level_interval_width }) \\
\alpha_{j} \sim N\left(\mu_{\alpha_{j}}, \sigma_{\alpha_{j}}^{2}\right) \text { for group } j=1, \ldots, J \\
\alpha_{k} \sim N\left(\mu_{\alpha_{k}}, \sigma_{\alpha_{k}}^{2}\right) \text { for participant } k=1, \ldots, K \\
\alpha_{l} \sim N\left(\mu_{\alpha_{l}}, \sigma_{\alpha_{l}}^{2}\right) \text { for claim } l=1, \ldots, L
\end{gathered}
$$

$$
\begin{gathered}
\text { Round_2_absolute_error } i \sim N\left(\mu, \sigma^{2}\right) \\
\mu=\alpha_{j[i], k[i], l[i]}+\beta_{1}(\text { claim_level_expertise })+\beta_{2}(\text { Round_2_claim_level_understanding }) \\
+\beta_{3}(\text { Round_2_claim_level_interval_width }) \\
\alpha_{j} \sim N\left(\mu_{\alpha_{j}}, \sigma_{\alpha_{j}}^{2}\right) \text { for group } j=1, \ldots, J \\
\alpha_{k} \sim N\left(\mu_{\alpha_{k}}, \sigma_{\alpha_{k}}^{2}\right) \text { for participant } k=1, \ldots, K \\
\alpha_{l} \sim N\left(\mu_{\alpha_{l}}, \sigma_{\alpha_{l}}^{2}\right) \text { for claim } l=1, \ldots, L
\end{gathered}
$$

Exploratory Question 3: Do those with lower confidence across claims (signalled by (i) lower average claim expertise ratings, (ii) lower average claim understanding ratings) improve more after discussion? (become more accurate) (at the participant level).

We explore this question using a linear mixed model of the difference in participants' Brier Scores between rounds as a function of the mean of their expertise across all claims they assessed as well as the mean understanding across all claims. We include a random-effect accounting for grouplevel variation (Equation 10). 


$$
\begin{gathered}
\text { Improvement }_{\text {Brier_Score }_{i}} \sim N\left(\alpha_{j[i]}+\beta_{1}(\text { mean_expertise })+\right. \\
\left.\beta_{2}(\text { mean_understanding }), \sigma^{2}\right) \\
\alpha_{j} \sim N\left(\mu_{\alpha_{j}}, \sigma_{\alpha_{j}}^{2}\right) \text { for group } j=1, \ldots, J
\end{gathered}
$$

\section{Qualitative analysis}

As described above, participants entered free-text responses into the platform to support their judgements. A total of 1891 qualitative justifications were provided by participants to contextualise their quantitative ratings. Three qualitative analysts independently coded each of these responses, interpreted within the available context of the participant's, and their group's responses, using a 'Known-Outcome' codebook that specified inclusion and exclusion criteria for 24 primary analytic categories, including codes that highlighted characteristics of reasoning about both the study design (e.g., sampling practices) and contextual considerations (e.g., journal reputation).

The 'Known-Outcome Codebook' was developed prior to the start of analysis, and was adapted from the repliCATS Reasoning Analysis Codebook that was developed for analysing SCORE claims (where replication outcomes were unknown). These codebooks were developed primarily by the two core members of the repliCATS Reasoning Analysis team (who did not participate in the coding of Known-Outcome claims) with input from other authors. Details of the iterative process involved in developing codebooks for the contextualised content analysis of SCORE claims have been described previously (Fraser et al. 2021; Hanea et al. 2021). Codes were selected from the larger codebook for inclusion within the smaller Known-Outcome Codebook on the basis of categories of greatest interest to the research team and considered specifically relevant to known-outcome claims.

The three qualitative analysts applied the Known-Outcome Codebook, to the free-text responses using computer-aided qualitative analysis software, NVivo (QSR International Pty Ltd 2018). The analytic process involved several steps. After codebook development, analysts undertook training sessions in the use of the codebook, and commenced coding the data. Following an initial coding of the data, inter-code reliability (ICR) scores were calculated. At this stage, codes that had met a pre-specified minimum ICR threshold (0.66) were used for analysis in another paper (Hanea et al. 2021).

For the mixed methods analysis within this paper, an additional step was undertaken. Analysts met collectively with the core Reasoning Analysis Team for calibration practices to cultivate consistency between each of the analysts on the relevant datasets. After further calibration (still in progress), each analyst will re-code the free-text responses using the Known-Outcome Codebook. Those responses consistently coded to an analytic category by multiple analysts will then be examined in relation to relevant mixed methods research questions (forthcoming).

The initial round of coding gives some indication of the types of responses provided by participants when they reason about replicability. They serve as placeholder examples to contextualise the 
quantitative analyses contained in this paper. Throughout the results section, coded justifications are drawn out to illustrate the context within which participants' individual and group quantitative judgements can be understood. As the qualitative analysis for this study is ongoing, only individual responses are used to further contextualise quantitative judgements, for example, to describe participant's self-reported reasoning for shifting their estimates or to illustrate the role of discussion in informing Round 2 judgements.

\section{Results and Discussion}

The aggregated (average) judgement achieved 84\% classification accuracy when predicting the replicability of social and behavioural science research claims. That is, for the claims participants evaluated, the average judgement was $>0.5$ probability on those that successfully replicated, and $<0.5$ to those that didn't $84 \%$ of the time (Figure 3 summarises participant judgements per claim).

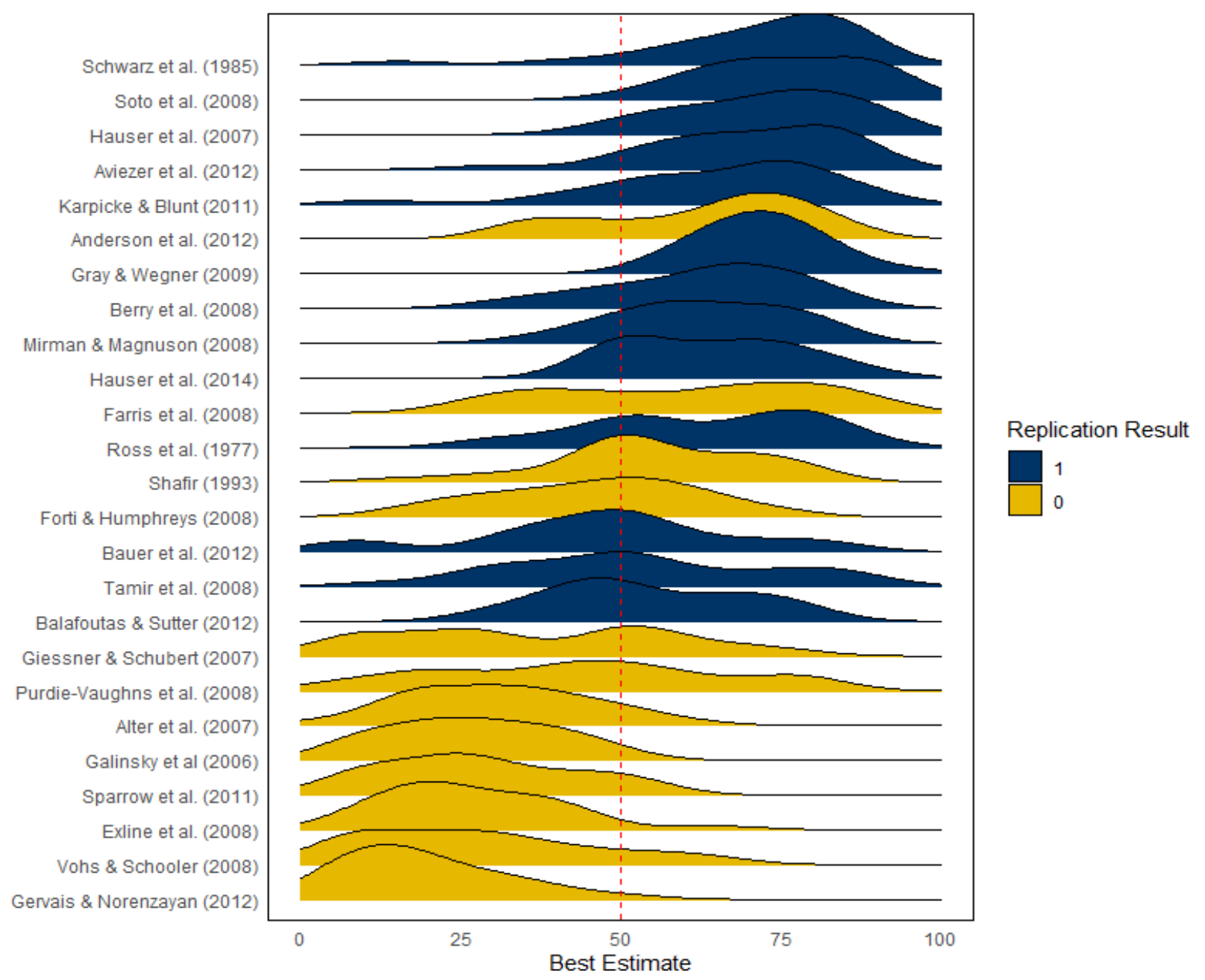

Figure 3. Smoothed distribution of participants' best estimates of replicability for each of the 25 research claims (studies), ordered by descending mean best estimates. Claims that successfully replicated are shown in blue, and claims that did not successfully replicate are shown in yellow. Best estimates greater than $50 \%$ indicate that the participant predicted the claim would successfully replicate, and less than $50 \%$ indicates that the participant predicted the claim would fail to replicate. 
Below, we present our quantitative results and some interpretation of our model outputs. Please note a caveat about interpreting data from these models, particularly those using responses of our 1-7 ordinal scales (i.e., claim level expertise and claim level understanding). This analysis assumes that everyone has interpreted the response scale in the same way, e.g., that a 7 for one person means the same as a 7 for someone else. In addition to the preregistered modelling reported here, we separately performed planned comparisons and correlation analyses, and results were consistent (to be reported in forthcoming supplementary materials).

\section{Hypothesis 1: Round 2 judgements (post-discussion) will be more accurate than Round 1 judgements (pre-discussion) (at the participant level)}

Average Brier Scores (error) were lower in Round 2 (after discussion) than in Round 1 (before discussion), suggesting that forecast revision in light of feedback and discussion improves accuracy (Figure 4). Twenty out of 25 participants were more accurate in Round 2, averaged across 25 claims.

a)

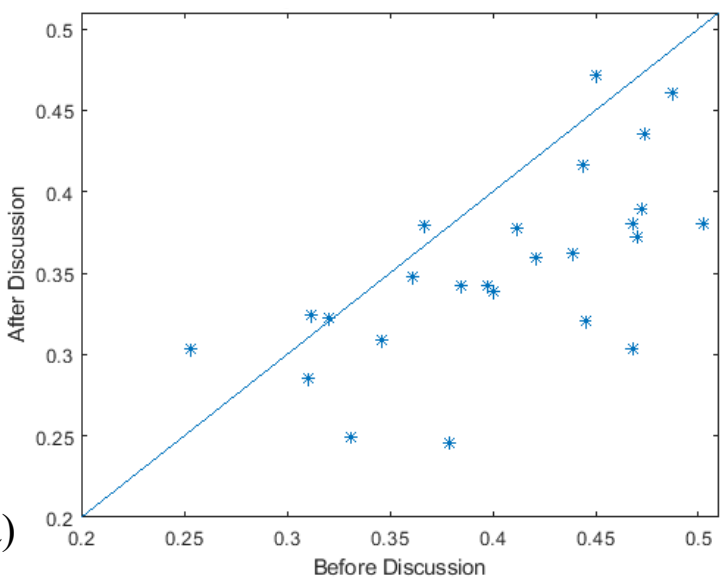

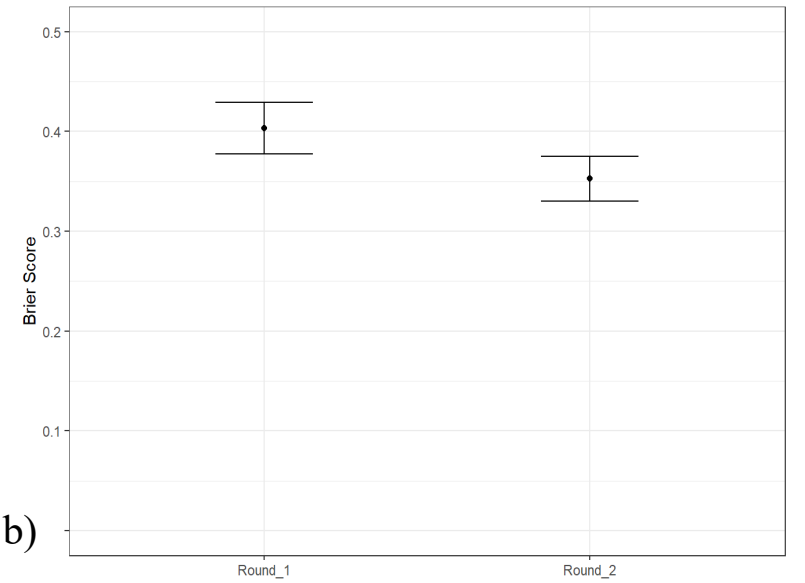

b) Figure 4. Average Brier Scores per participant were lower (more accurate) in Round 2, after discussion, than
in Round 1, before discussion. This is indicated by the majority of the 25 datapoints being below the identity line (a), and the lack of overlapping $95 \%$ confidence intervals between rounds (b).

Visual assessment of the confidence intervals ('inference by eye') suggests a statistically significant difference in overall accuracy before and after discussion, since the confidence intervals do not overlap. The paired t-test confirms a statistically significant difference in accuracy (Brier score) between rounds of 0.05 [0.03, 0.07], $\mathrm{p}<0.001$.

In additional exploratory analysis, we found that twice as many best estimate judgements improved (254) from Round 1 to Round 2 as got worse (121), while 250 stayed the same (i.e. didn't change). Those that improved in Round 2 shifted on average 13\% points, while those that got worse shifted $11 \%$ points. 
There were only three cases of people shifting dramatically after discussion (50\% points or more). All were more accurate in Round 2. These large shifts were contextualised by the individuals and expectedly noted that discussion had prompted their re-evaluation. For example, one participant who shifted their best estimate from 25 to 90 noted in Round 1 that their low estimate was informed by a reported effect size that was "unbelievably large" and the use of a rare inferential test which "raises a red flag". Their shift in Round 2 was briefly explained as follows:

\section{Group was really positive about the study which led me to believe I am being overly critical ... Not everyone is as skeptical as me, and there are no clear red flags}

In the second dramatic shift - a reduction of best estimate from 60 to 10 - the individual noted in their reasoning in Round 2 that discussion included reputational aspects of the disciplinary domain as well as the study itself, noting that "the effect seems large for priming", and that "some of [the author's] work hasn't replicated and is really underpowered". The discussion of the third dramatic shift showed that members of the group had private knowledge of the authors' work which was reflected in Round 2 reasoning in the group. Overall, although these three individuals all shifted in the 'right' direction, this is clearly not enough data to draw conclusions from.

\section{Hypothesis 2: Those who perform well on a quiz testing familiarity with previous research and concepts related to assessments of replicability will be more accurate on the main task (at the participant level).}

These models indicate that in Round 1, participants overall quiz scores had a non-significant near zero effect on accuracy, and groups have near zero effect. In Round 2, quiz score has a statistically significant $(p=0.0017)$ effect on accuracy (Figure 5), but of a small magnitude (each correct answer on the quiz drops the participant's Average Brier Score by $\sim 0.01[-0.02,-0.005])$. Again, groups have near zero effect on accuracy.

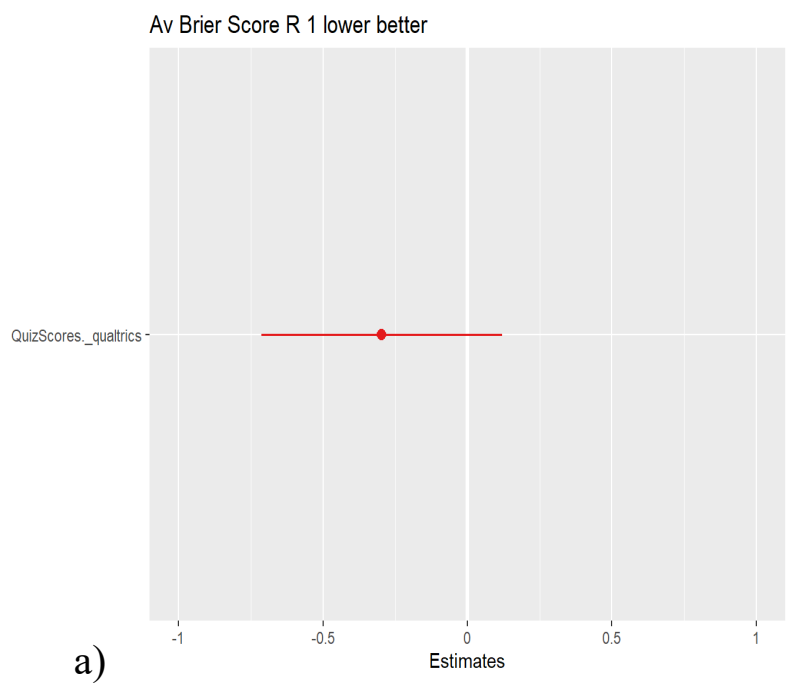

a)

Figure 5. Standardized model coefficient with $95 \%$ CI showing the relationship between Quiz scores and Average Brier scores per participant for Round 1, before discussion (a) and Round 2, after discussion (b). 
In additional exploratory analysis examining the two main components of the quiz separately, it appears that performance on the statistical concepts component (out of 12 possible points) carries the most weight in any possible relationship with accuracy (Figure 6), with a weak correlation in Round 1 (Spearman's $\rho=-0.356$ [-0.666, 0.058]), and a stronger correlation in Round 2 (Spearman's $\rho=-0.516[-0.768,-0.125]$ ). The meta-research knowledge component (out of 10 possible points) had non-significant, near zero correlations with accuracy in both rounds. With hindsight, this might be because items in the statistical component part of the quiz requiring statistical thinking or reasoning that can be more readily applied to claim evaluation. The metaresearch items, on the other hand, target participants' memory of published replication success rates or average statistical power from previous metaresearch projects. Whilst knowledge of those base-rates arguably help form informative priors for participants' judgements, they do not discriminate performance as well as statistical reasoning.

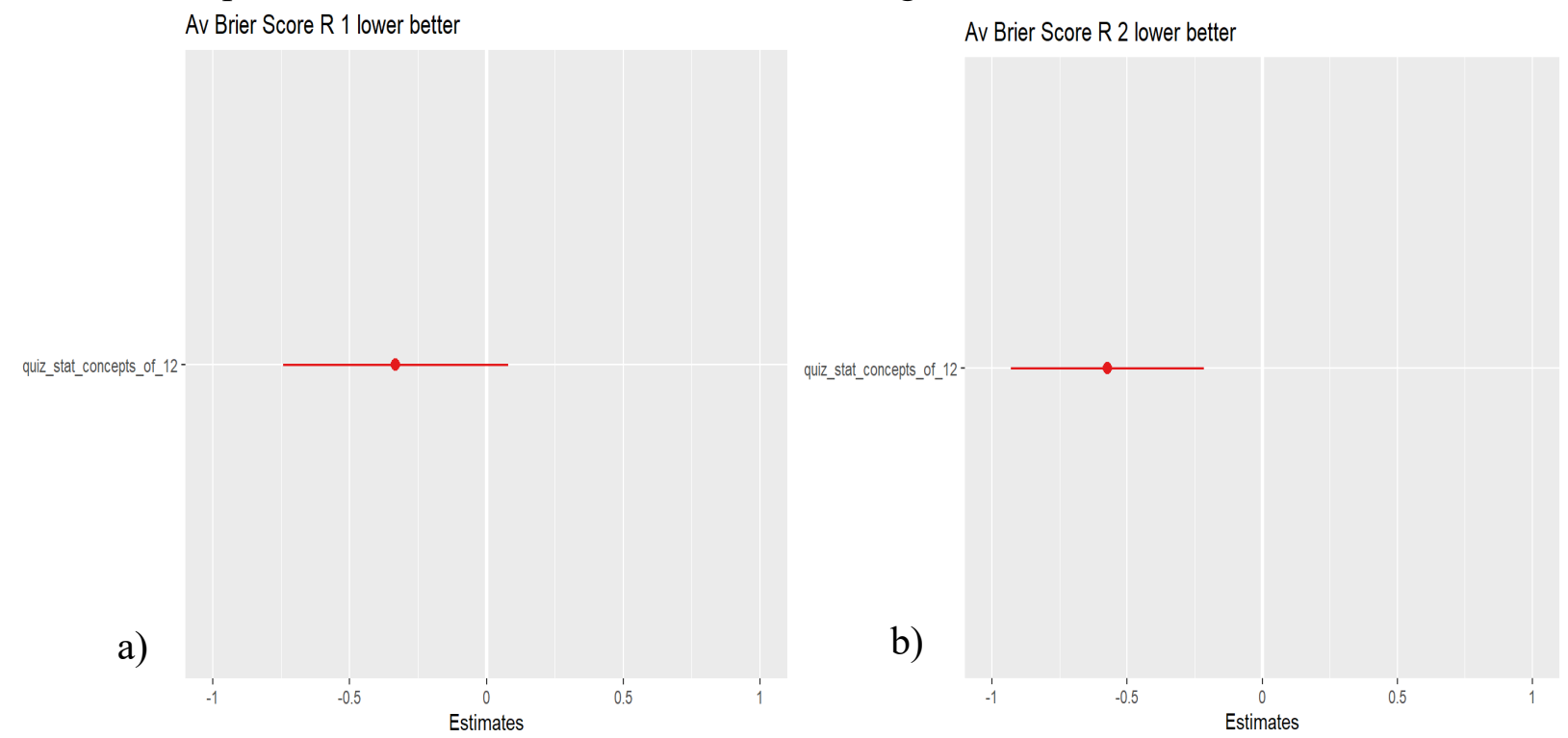

Figure 6. Relationship between participant scores on the statistical knowledge component of the quiz and accuracy in Round 1 (a) and Round 2 (b).

Exploratory Question 1: What are the demographic, experience and psychometric characteristics of (a) more accurate judges, and (b) those who update their judgements most between rounds?

As anticipated, we did not detect many meaningful or statistically significant relationships between demographics, experience and training variables with judgement performance. Here, we present our preliminary results from Spearman rank correlation analyses.

Experience with preregistration vs accuracy: Those who have experience with preregistration were more accurate in Round $1(\mathrm{p}=0.01)$ and Round $2(\mathrm{p}=0.001)$ (Figure 7). 

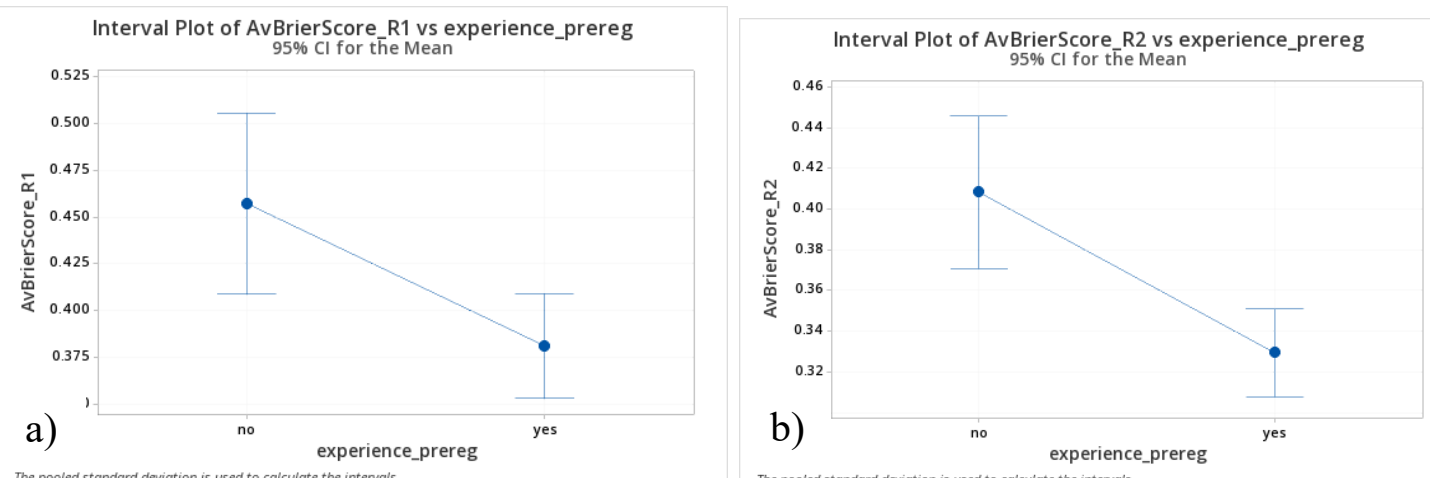

Figure 7. Relationship between participants' experience with preregistration and average Brier Score error (inverse of accuracy, lower is better), in Round 1 (a) and Round 2 (b). Error bars indicate 95\% confidence intervals.

Gender vs accuracy: Gender has a statistically significant relationship with accuracy $(\mathrm{p}=0.012)$ in Round 1 (males more accurate) but in not in Round 2.
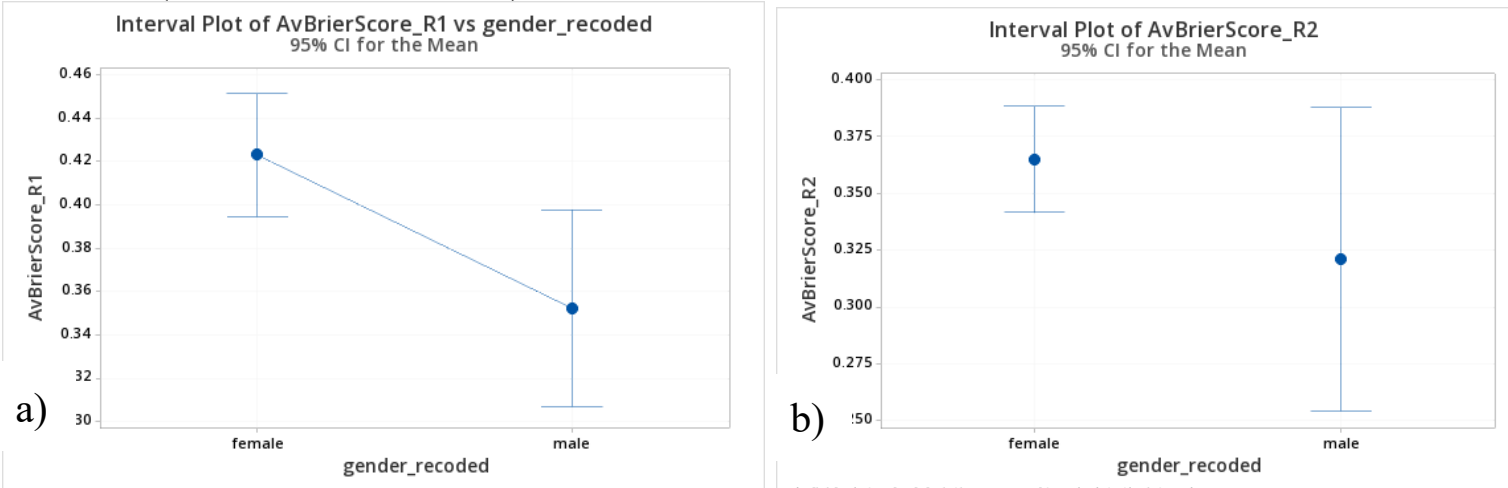

Figure 8. Relationship between participants' gender and average Brier Score error (inverse of accuracy, lower is better), in Round 1 (a) and Round 2 (b). Error bars indicate 95\% confidence intervals.

Statistics and methods training $v$ accuracy: There may be a relationship between statistical training and accuracy, with those who had completed fewer statistics and methods courses performing marginally worse than those who had completed more, but the $95 \%$ is CI sitting on zero, for both Rounds 1 and 2, and our capped response option at 6+ courses makes this difficult to interpret.
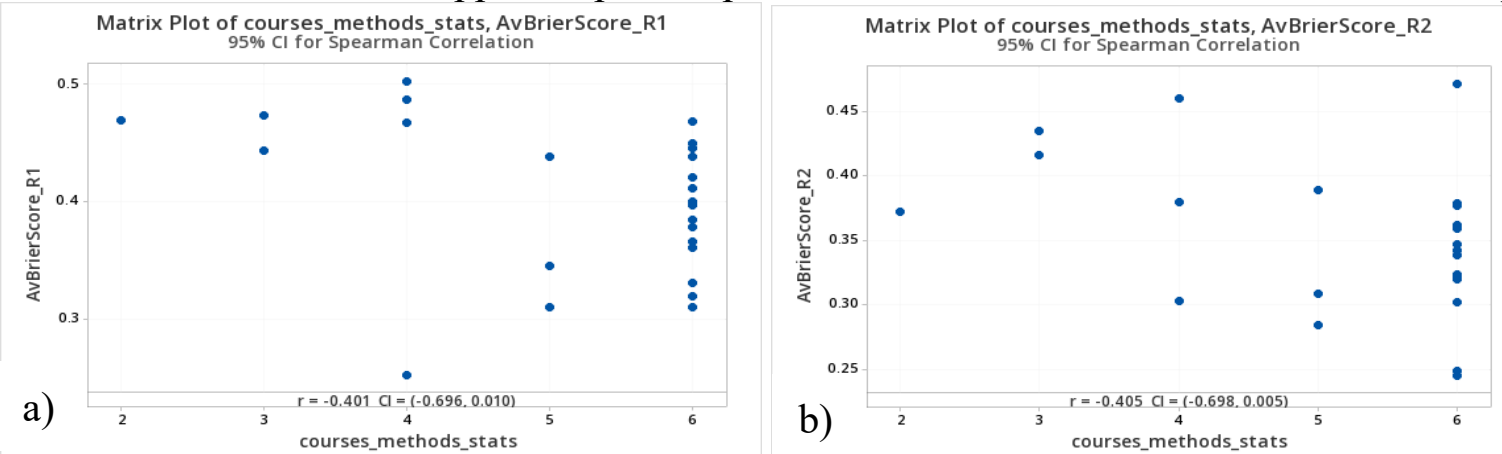

Figure 9. Relationship between number of courses the participant had taken in research methods and / or statistics and their average Brier Score error (inverse of accuracy, lower is better), in Round 1 (a) and Round 2 (b). 
AOT / CRT / Berlin Numeracy v accuracy and updating: Results from standardised tests that were administered to participants, i.e. the Cognitive Reflection Test (CRT), Berlin Numeracy (BN), and the Actively Open-minded Thinking test (AOT), also did not yield particularly strong results. Nonetheless, the relationships we do see are as expected. There was a small statistically significant relationship between Berlin Numeracy and accuracy, i.e. Average Brier Scores in Round 1. Recall that Brier scores are a measure of judgment error, so a negative correlation coefficient here indicates that as numeracy goes up, error goes down (Spearman's $\rho=-0.42[-0.78,0.04], p=0.04$ ). We also saw statistically significant relationships between the two numeracy -like scales (CRT and BN, Spearman's $\rho=0.45[0.14,0.75], p=0.03$ ), and between CRT and shifting (Spearman's $\rho=0.47[0.02,0.72], p=0.02$ ). Results from the AOT test lacked variation (everyone scored highly, i.e., as 'actively open minded'). Accordingly, we did not detect any relationships involving that variable.

Exploratory Question 2: Are those who update their judgements between rounds more accurate in Round 2 (after discussion, across all claims)?

Those who shift the most on average not only improve the most between rounds (not surprisingly, assuming they are shifting towards the correct answer)(Spearman's $\rho=0.50[0.11,0.76])$, but they are also the most accurate in Round 2 (lower Brier score error, on average) (Figure 10).

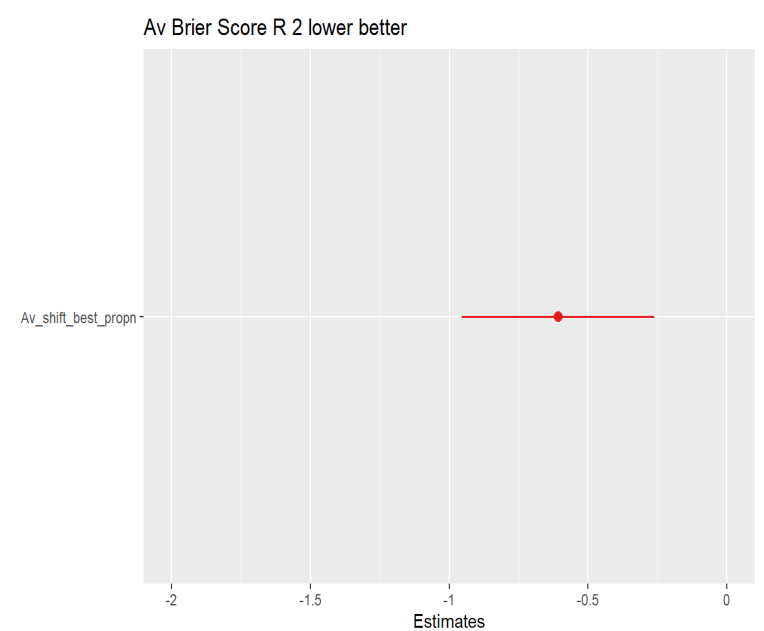

Figure 10. Standardized model coefficient with $95 \%$ CI showing the relationship between participants' updating after discussion (average shift), and average Brier score error (inverse of accuracy) in Round 2.

Hypothesis 3: Those who update (shift) their estimates most between rounds will be less confident in their judgements on average, as indicated by: (i) lower mean claim-level expertise ratings, (ii) lower mean claim-level understanding ratings (at the participant level).

(i) Claim level expertise: We detected that when expertise ratings are higher, the amount that a participant shifts their best estimate judgements after discussion is reduced. This is a statistically significant effect ( $p=0.036$ ), of a small magnitude. Each unit increase in average expertise (on the 1-7 scale) reduces the participant's average shift by $\sim 1.025[-1.90,-0.12]$ (on a $0-100$ scale, but ranging from 3-15). We detected a smaller and non significant difference in the same direction for interval width shifting. 
(ii) Claim level understanding: Understanding ratings were associated with inconsistent effects on the extent of shifting, with statistically non significant effects for both best estimate and interval width. We refrain from interpreting these results further as the variation in understanding responses is too small to properly investigate this question. Everyone appeared to have a good understanding of the claims, ranging from 4.9 to 6.7 in Round 1, and 5.3 to 6.9 for Round 2 (on a scale of 1-7). In hindsight, this reflects our decision to select the claims on the basis that they were reasonably straightforward and not confusing to participants. Therefore, it is not surprising that we found no meaningful relationships with this and other variables.

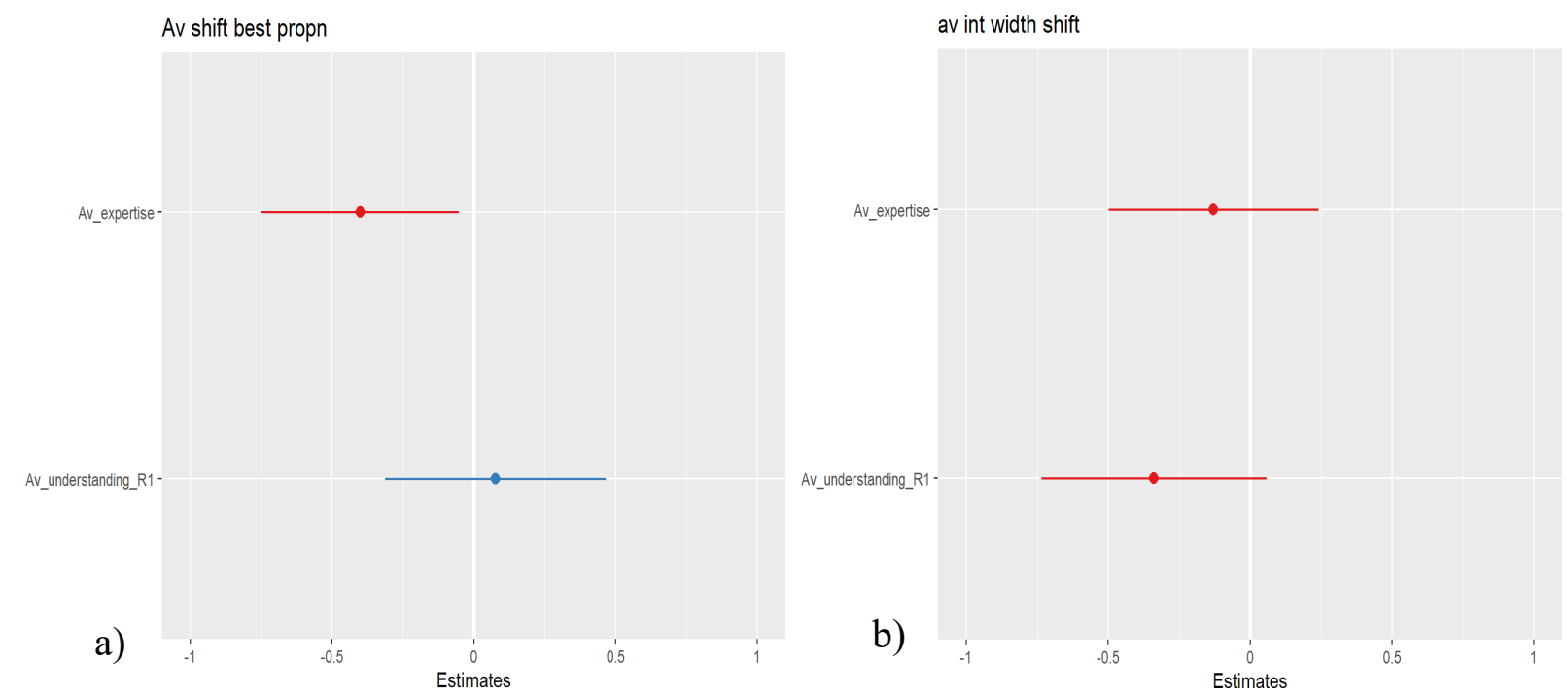

Figure 11. Standardized model coefficients with $95 \%$ CIs showing the relationship between average expertise, average understanding and average size of the shift per participant between rounds, after discussion, showing their change in best estimates (a) and change in interval widths (b).

The following examples of qualitative reasoning help contextualise the different roles discussion played in influencing participant confidence and updating between rounds in this study. In the first example, one individual shifted their best estimate of replicability from 55 to 25 , noting that:

\section{Discussion strengthened my beliefs about p-hacking, lack of pre-study planning, selective reporting and other QRPS. I find the underlying premise unlikely and anticipate there are a number of methodological issues}

In this example it is clear that discussion improved the individual's confidence in their original assessment of the claim. Another individual analysing the same claim also shifted their best estimate from 75 to 40 , noting instead that discussion highlighted aspects of the claim they had not fully considered:

During the discussion, I heard ... some counter-evidence for the premise of the study. Also, I learned that they [the authors] used an existing data set, and ran multiple tests, etc. All these got me worried a bit. The stats still looks good to me but I lowered my estimates in general.

The final example included here illustrates the most common role of discussion in correcting or clarifying the claim being evaluated. This individual updated their best estimate from 70 to 50 
between rounds, explaining that discussion clarified a critical aspect of the study design and operationalisation:

... So I missed a very important point while making my first judgment. I think I
now understand the claim better after the discussion. There was a mismatch
between the statistics (sample size vs. reported df) and I did not realized [sic]
this at the beginning. Small sample size but strong effects, I still think that this
might replicate but I also accept that there is good chance that it won't.

Hypothesis 4: Judgements will be more accurate when associated with signals of higher confidence on those particular judgements, specifically, (i) higher claim expertise ratings, (ii) higher claim understanding ratings, (iii) more certain (narrower) intervals.

We found a small but not quite statistically significant $(p=0.054)$ improvement in accuracy with higher expertise ratings. We found a larger and statistically significant effect $(p<0.001)$ effect of uncertainty on accuracy, where wider intervals (i.e., less certain intervals) are associated with decreased accuracy (Figure 12). So, at a judgement level, in Round 1, a 10\% increment difference in interval width would suggest a best estimate score that is $\sim 2.4 \%[1.3,3.4]$ (on the $0-100$ scale) further away from the (correct) certainty limit. And as above in Hypothesis 3, we found no statistically significant or meaningful effect of 'understanding' ratings (in Figure 12, 'comprehension') on accuracy, but see above for our concern over lack of variation in this measure. On the whole, interval width seems to provide a better measure of confidence/uncertainty than other self ratings of confidence, i.e., expertise and understanding, even when the latter are elicited at specific claim level like interval width. This seems to be the case even though we think interval width likely to tell us more in quantitative than probability judgements.

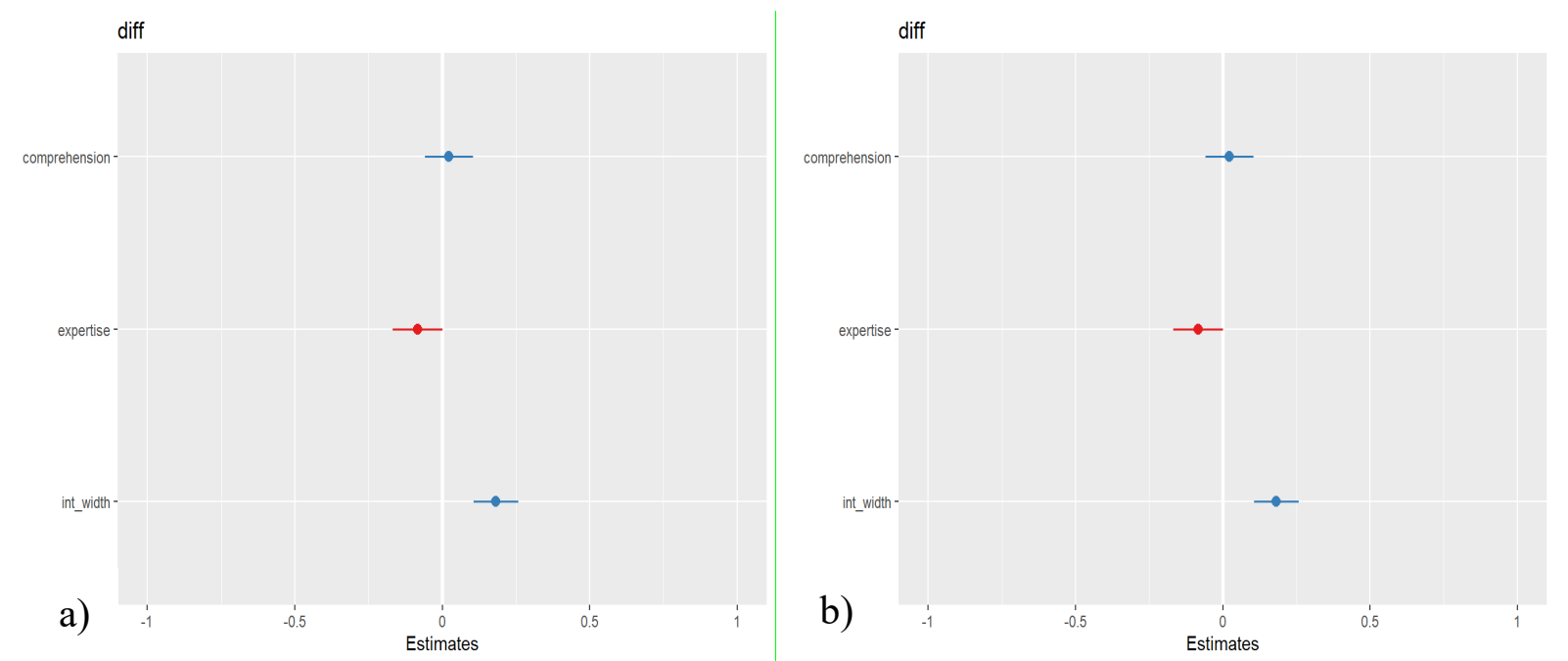

Figure 12. Standardized model coefficient with $95 \%$ CI showing the relationship between understanding (here, "comprehension") scores, expertise scores, and judgement-level error (here, "diff", i.e. absolute difference between outcome and best estimate, inverse of accuracy), in Round 1, before discussion (a) and Round 2 , after discussion (b). 
Below are a variety of examples showing ways in participants' express confidence in their estimates, discussing notions of expertise, certainty and understanding. In the first example below, the participant is explicitly clear that the increased certainty they felt following discussion had been translated into narrower bounds in Round 2:

I still don't think it will replicate because it is a very small effect (thought it would be nice to see what happens with an adequate sample size), though I moved my estimate up a bit to account for my better understanding of the proposed mechanism. But I feel less uncertain after the discussion so my bounds are smaller.

In other cases, participants expressed having been generally swayed towards another member(s) estimates:

Other people in the group had more knowledge in this area than me and they expressed some more confidence in the design so it raised my lower bound a bit.

I brought up my best and lower estimates after another group member explained the study to me better.

There were also several examples where participants' confidence had clearly been impacted by discussion but their comments did not explicitly identify how this was translated (or not) into updated estimates.

My group had a lot more information about this type of research, and it made me a little more confident in this effect (slightly stronger prior).

Really influential to hear another group members say she's observed this pattern multiple times in her own data.

For some, discussion reinforced participants' confidence in their own initial estimates.

the arguments of the rest of the group against the claim seemed to be mostly methodological criticism. but as the replication would be conducted using the same methodology I don't think these arguments speak against a successful replication. The arguments in favor of a successful replication pretty much agree with my own thoughts on this, so I feel more confident now.

\section{Exploratory Question 3: Do those with lower confidence across claims (signalled by (i) lower average claim expertise ratings, (ii) lower average claim understanding ratings) improve more after discussion? (become more accurate) (at the participant level).}

Additional analyses suggest that people with lower self-rated expertise (averaged per claim) improved the most between rounds (became more accurate, Figure 13). We did not find a statistically significant relationship between self-rated claim understanding (averaged per claim) and improvement. 

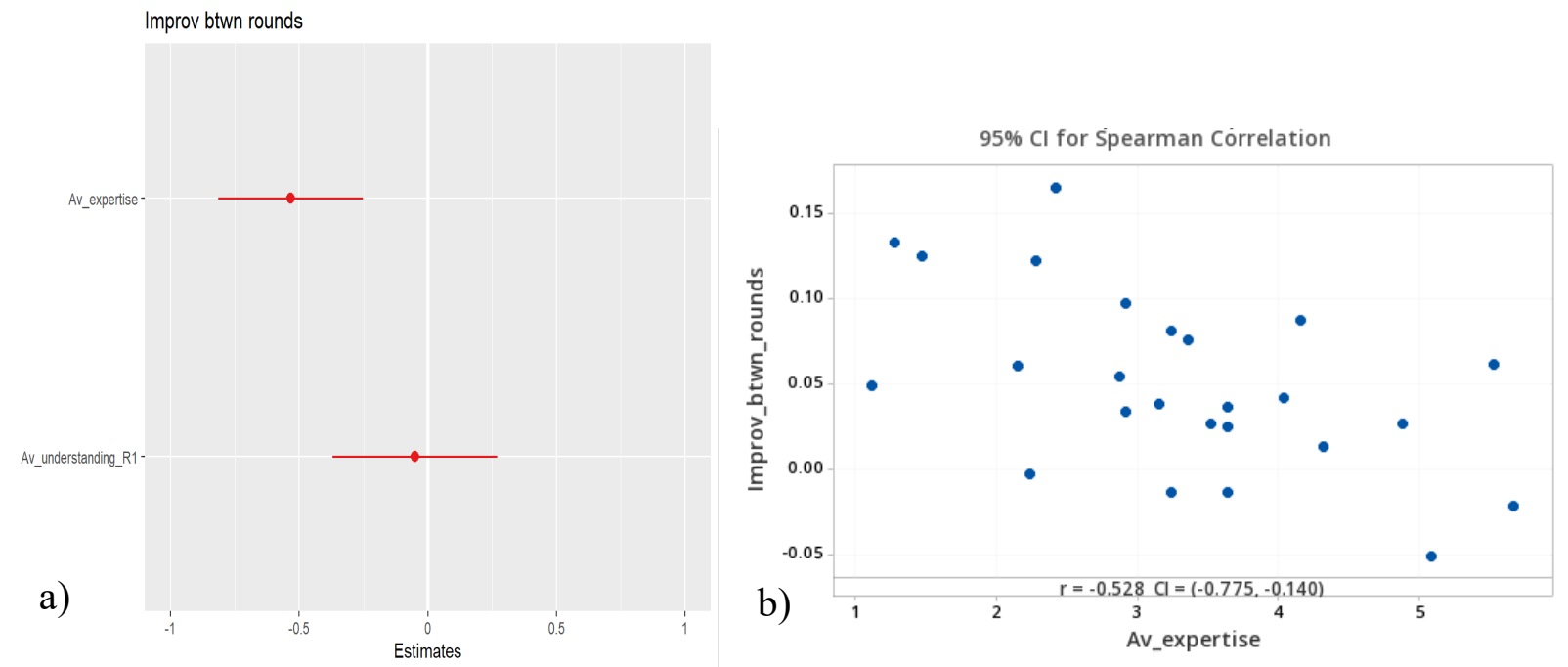

Figure 13. Participants with lower self-rated expertise improved their accuracy the most between rounds, after discussion (i.e. they had lower Average Brier Scores in Round 2). a) Standardized model coefficients with 95\% CIs showing the relationship between average expertise, average understanding and average accuracy improvement between rounds per participant, after discussion, and b) scatterplot of improvement vs average self-rated expertise.

\section{General Discussion}

Compared with the results of similar prediction markets and surveys, participants in our study were very good at predicting the replicability research claims from Social and Behavioural Sciences. Our prediction accuracy for these 25 studies was $84 \%$, and prediction markets for the studies our claims were sampled from ranged from $71 \%-86 \%$. (Surveys of the same studies ranged 58\%-86\%). The large range in prediction market and survey results in part reflects changes in the methodology of conducting replication studies themselves. In earlier projects (e.g., Reproducibility Project: Psychology), replication studies were on average not as high-powered as replication studies in more recent projects (Social Science Reproducibility Project). The set of claims we evaluated here span both earlier and more recent projects, but our sample only included claims for evaluation that were tested against a high-powered replication study (at least $90 \%$ power to detect an effect $75 \%$ of size of that seen in the original study). This sampling strategy may explain why our own highly accurate results are more comparable to those achieved in later replication prediction projects (e.g., (e.g., Camerer et al. 2018; Forsell et al. 2019) than earlier ones (e.g., Dreber et al. 2015).

We acknowledge the small sample size of our study. This is a common shortcoming of studies involving groups. We also acknowledge that our participants were making "predictions" restropectively, so there is a possibility they were aware of the published outcomes, although see our Methods for how we attempted to buffer against this possibility. Nonetheless, the high accuracy levels in our results support the idea that structured elicitation methods, like the IDEA protocol, have a useful role to play in the evaluation of research. 


\section{Effect of updating and discussion on replicability judgements}

The improvement we found in Round 2, following feedback and discussion, is comparable to previous studies that used the structured IDEA protocol to elicit judgements. In our study, $80 \%$ of individuals moved towards the correct answer following feedback and discussion (Round 2). In Hemming et al. (2018b), individuals moved towards the correct answer $67 \%$ of the time, and when they did, it was usually a meaningful difference. In contrast, those who moved away from the correct answer generally only did so by a small amount. Our results are also compatible with those of the Good Judgement Project (Mellers et al. 2015a), who found that those who worked in teams, discussed and debated evidence and exchanged rationales were more accurate than those who worked alone.

\section{Participant expertise and prior knowledge}

As discussed above in Results: Hypothesis 2, the overall quiz score did not serve as a useful predictor of accuracy. Having said that, exploratory analysis suggests more of a signal from the statistical concepts component of the quiz than the metaresearch component. This is something we will investigate in future research.

We also reported on the very limited predictive power of more traditional markers of expertise (i.e. background, education, publications, training and experience), again in exploratory analysis. This (lack of) result was unsurprising, and is in line with other research that has also failed to detect relationships here (Burgman et al. 2011b; Burgman 2015).

Given the persistent challenges in determining, a priori, who are the best experts for forecasting and judgements, it was notable to find a statistically significant relationship between accuracy and one marker of expertise, namely experience with preregistration. Those who reported that they had "preregistered at least one study" were statistically significantly more accurate, in both Round 1 and Round 2. It is potentially useful to know that practical experience with preregistering research in advance, i.e., thinking through and transparently documenting the design and analysis plan for a study, may indicate an ability to accurately evaluate the quality (in this case, replicability) of other published research. Of course, this relationship may well disappear as preregistration becomes more widespread. It is perhaps only indicative now because we are still in an 'early adopters' phase.

\section{The relationship between confidence and accuracy}

In this study, we explored the relationship between subjective confidence or uncertainty and accuracy in three different ways. For each claim, a) participants rated their expertise in the domain of the claim, b) their understanding of the claim, and c) they provided uncertainty in the form of interval bounds. We report above (Hypothesis 4) that higher expertise self ratings for the specific claim domain were weakly associated with more accurate predictions. This is in the direction we would expect but more research is needed to substantiate the relationship. We did not detect a relationship between claim level understanding and accuracy. We did, however, find a relationship between interval width and accuracy at the judgement level, where best estimates accompanied by narrower intervals were more accurate than those accompanied by wider (less certain) intervals. This relationship has been previously noted when eliciting intervals around quantities with a given 
level of confidence (e.g., Yaniv 1997; Yaniv \& Foster 1997). This makes more conceptual sense in unbounded quantity estimates than in the probability judgements we have elicited in this study, because probabilistic best estimates closer to the extremes ( 0 or 1$)$ already contain information about subjective confidence. In addition, a more extreme probability judgement is inherently more likely to be accompanied by a narrower interval due to the smaller distance to the nearest certainty limit, unless it is highly asymmetric.

\section{Conclusion}

The repliCATS structured protocol elicited highly accurate predictions of replicability, comparable to the best results previously seen in prediction markets. Our approach also elicited detailed information about the reasoning and justifications participants gave for their judgements, which provides insight into how to further improve predictions and evaluation of research in future. For example, successful reasoning strategies may inform peer review training, and be incorporated into future evaluation rubrics or checklists. The opportunity to receive feedback from the group, share information and update judgements are the critical components of our repliCATS approach, and contributed to the accuracy of predictions in this study. We expect accuracy of predictions to improve in the future, as elicitation methods are refined, and as the methodology and power of replication studies improve.

\section{Acknowledgements}

This project is sponsored by the Defense Advanced Research Projects Agency (DARPA) under cooperative agreement No. HR001118S0047. The content of the information does not necessarily reflect the position or the policy of the Government, and no official endorsement should be inferred.

\section{References}

Bahrami B, Olsen K, Latham PE, Roepstorff A, Rees G, Frith CD. 2010. Optimally interacting minds. Science 329:1081-1085.

Bates D, Maechler M, Bolker B, Walker S. 2015. Fitting Linear Mixed-Effects Models Using lme4. Journal of Statistical Software 67:1-48.

Begley CG, Ellis LM. 2012. Drug development: Raise standards for preclinical cancer research. Nature 483:531-533.

Benjamin D, Mandel DR, Kimmelman J. 2017. Can cancer researchers accurately judge whether preclinical reports will reproduce? PLOS Biology 15:e2002212.

Brier GW. 1950. Verification of forecasts expressed in terms of probability. Monthly Weather Review 78.

Burgman M, Carr A, Godden L, Gregory R, McBride M, Flander L, Maguire L. 2011a. Redefining expertise and improving ecological judgment. Conservation Letters 4:81-87.

Burgman MA 2015. Trusting Judgements: How to Get the Best out of Experts. Cambridge University Press, Cambridge.

Burgman MA, McBride M, Ashton R, Speirs-Bridge A, Flander L, Wintle B, Fidler F, Rumpff L, Twardy C. 2011 b. Expert status and performance. Plos One 6:e22998. 
Camerer CF, et al. 2016. Evaluating replicability of laboratory experiments in economics. Science 351:1433-1436.

Camerer CF, et al. 2018. Evaluating the replicability of social science experiments in Nature and Science between 2010 and 2015. Nature Human Behaviour 2:637.

Cokely ET, Galesic M, Schulz E, Ghazal S, Garcia-Retamero R. 2012. Measuring risk literacy: The Berlin Numeracy Test. Judgment and Decision Making 7:25-47.

Dana J, Atanasov P, Tetlock P, Mellers B. 2019. Are markets more accurate than polls? The surprising informational value of "just asking". Judgment \& Decision Making 14:135-147.

Dreber A, Pfeiffer T, Almenberg J, Isaksson S, Wilson B, Chen Y, Nosek BA, Johannesson M. 2015. Using prediction markets to estimate the reproducibility of scientific research. Proceedings of the National Academy of Sciences 112:15343-15347.

Ebersole CR, et al. 2016. Many Labs 3: Evaluating participant pool quality across the academic semester via replication. Journal of Experimental Social Psychology 67:68-82.

Errington TM, Iorns E, Gunn W, Tan FE, Lomax J, Nosek BA. 2014. An open investigation of the reproducibility of cancer biology research. eLife 3 .

Farrell S. 2011. Social influence benefits the wisdom of individuals in the crowd. Proceedings of the National Academy of Sciences of the United States of America 108:E625-E625.

Forsell E, Viganola D, Pfeiffer T, Almenberg J, Wilson B, Chen Y, Nosek BA, Johannesson M, Dreber A. 2019. Predicting replication outcomes in the Many Labs 2 study. Journal of Economic Psychology 75:102117.

Fraser H, Bush M, Wintle B, Mody F, Smith ET, Hanea A, ..., Fidler F. 2021. Predicting reliability through structured expert elicitation with repliCATS (Collaborative Assessments for Trustworthy Science). https://doi.org/10.31222/osf.io/2pczv Preprint, February 22.

Frederick S. 2005. Cognitive Reflection and Decision Making. Journal of Economic Perspectives 19:25-42.

French S. 2011. Aggregating expert judgement. Revista de la Real Academia de Ciencias Exactas Fisicas y Naturales Serie a-Matematicas 105:181-206.

Galinsky A, D., Kray L, J. 2003. From thinking about what might have been to sharing what we know: The effects of counterfactual mind-sets on information sharing in groups. Journal of Experimental Social Psychology 40:606-618.

Hamilton DG, Fraser H, Hoekstra R, Fidler F. 2020. Journal policies and editors' opinions on peer review. eLife 9:e62529.

Hanea A, Wilkinson DP, McBride M, Lyon A, van Ravenzwaaij D, Singleton Thorn F, ..., Wintle BC. 2021. Mathematically aggregating experts' predictions of possible futures. https://doi.org/10.31222/osf.io/rxmh7 Preprint, February 23.

Hanea AM, McBride M, Burgman MA, Wintle BC, Fidler F, Flander L, Twardy CR, Manning B, Mascaro S. 2017. Investigate Discuss Estimate Aggregate for structured expert judgement. International Journal of Forecasting 33:267-279.

Hemming V, Armstrong N, Burgman MA, Hanea AM. 2020. Improving expert forecasts in reliability: Application and evidence for structured elicitation protocols. Quality and Reliability Engineering International:623.

Hemming V, Burgman MA, Hanea AM, McBride MF, Wintle BC. 2018a. A practical guide to structured expert elicitation using the IDEA protocol. Methods in Ecology and Evolution 9:169-181. 
Hemming V, Walshe TV, Hanea AM, Fidler F, Burgman MA. 2018b. Eliciting improved quantitative judgements using the IDEA protocol: A case study in natural resource management. PLoS ONE.

Hoogeveen S, Sarafoglou A, Wagenmakers E-J. 2020. Laypeople Can Predict Which SocialScience Studies Will Be Replicated Successfully. Advances in Methods and Practices in Psychological Science:2515245920919667.

Isager PM, et al. 2020. Deciding what to replicate: A formal definition of "replication value" and a decision model for replication study selection. Preprint https://doi.org/10.31222/osf.io/2gurz.

Janis IL 1982. Groupthink: Psychological Studies of Policy Decisions and Fiascoes. Houghton Mifflin, Boston.

Kelly CD. 2006. Replicating empirical research in behavioral ecology: how and why it should be done but rarely ever is. The Quarterly Review Of Biology 81:221-236.

Klein RA, et al. 2014. Investigating Variation in Replicability. Social Psychology 45:142-152.

Klein RA, et al. 2018. Many Labs 2: Investigating Variation in Replicability Across Samples and Settings. Advances in Methods and Practices in Psychological Science 1:443-490.

Koriat A. 2012. When are two heads better than one and why? Science 336:360-362.

Koriat A, Lichtenstein S, Fischhoff B. 1980. Reasons for confidence. Journal of Experimental Psychology-Human Learning and Memory 6:107-118.

Kuznetsova A, Brockhoff P, Christensen R. 2017. lmerTest Package: Tests in Linear Mixed Effects Models. Journal of Statistical Software 82:1-26.

Larrick RP, Soll JB. 2006. Intuitions about combining opinions: Misappreciation of the averaging principle. Management Science 52:111-127.

Lorenz J, Rauhut H, Schweitzer F, Helbing D. 2011. How social influence can undermine the wisdom of crowd effect. Proceedings of the National Academy of Sciences of the United States of America 108:9020-9025.

McBride M, et al. 2012. Structured elicitation of expert judgments for threatened species assessment: a case study on a continental scale using email. Methods in Ecology and Evolution 3:906-920.

Mellers B, Stone E, Atanasov P, Rohrbaugh N, Metz SE, Ungar L, Bishop MM, Horowitz M, Merkle E, Tetlock P. 2015a. The Psychology of Intelligence Analysis: Drivers of Prediction Accuracy in World Politics. Journal of Experimental Psychology: Applied 21:114.

Mellers B, et al. 2015b. Identifying and cultivating superforecasters as a method of improving probabilistic predictions. Perspectives On Psychological Science: A Journal Of The Association For Psychological Science 10:267-281.

Mellers B, et al. 2014. Psychological Strategies for Winning a Geopolitical Forecasting Tournament. Psychological Science 25:1106-1115.

Mojzisch A, Schulz-Hardt S. 2010. Knowing others' preferences degrades the quality of group decisions. Journal of Personality and Social Psychology 98:794-808.

Nosek BA, Bar-Anan Y. 2012. Scientific Utopia: I. Opening Scientific Communication. Psychological Inquiry 23:217-243.

Nosek BA, Errington TM. 2020. What is replication? PLOS Biology 18:e3000691.

Önkal D, Lawrence M, Sayım KZ. 2011. Influence of differentiated roles on group forecasting accuracy. International Journal of Forecasting 27:50-68. 
Open Science Collaboration. 2015. Estimating the reproducibility of psychological science. Science 349:aac4716.

Pearson R, et al. 2021. Eliciting Group Judgements about Replicability: A Technical Implementation of the IDEA Protocol. Proceedings of the 54th Hawaii International Conference on System Sciences, Hawaii.

Pinheiro J, Bates D, DebRoy S, Sarkar D, R Core Team. 2020. nlme: Linear and Nonlinear Mixed Effects Models.

Postmes T, Spears R, Cihangir S. 2001. Quality of decision making and group norms. Journal of Personality and Social Psychology 80:918-930.

QSR International Pty Ltd. 2018. NVivo qualitative data analysis software.

R Core Team. 2020. R: A language and environment for statistical computing. R Foundation for Statistical Computing, Vienna, Austri.

Schmidt S. 2009. Shall We Really Do It Again?: The Powerful Concept of Replication Is Neglected in the Social Sciences. Review of General Psychology 13:90-100.

Sniezek JA, Henry RA. 1990. Revision, weighting, and commitment in consensus group judgment. Organizational Behavior \& Human Decision Processes 45:66-84.

Soll JB, Klayman J. 2004. Overconfidence in interval estimates. Journal of Experimental Psychology: Learning Memory and Cognition 30:299-314.

Speirs-Bridge A, Fidler F, McBride M, Flander L, Cumming G, Burgman M. 2010. Reducing overconfidence in the interval judgments of experts. Risk Analysis 30:512-523.

Stanovich K, West R. 1997. Reasoning independently of prior belief and individual differences in actively open-minded thinking. Journal of Educational Psychology 89:342-357.

Stasser G, Titus W. 2003. Hidden profiles: A brief history. Psychological Inquiry 14:304-313.

Teigen KH, Jørgensen M. 2005. When 90\% confidence intervals are 50\% certain: On the credibility of credible intervals. Applied Cognitive Psychology 19:455-475.

Wintle BC, Fidler F, Vesk P, Moore J. 2013. Improving visual estimation through active feedback. Methods in Ecology and Evolution 4:53-62.

Yaniv I. 1997. Weighting and trimming: Heuristics for aggregating judgments under uncertainty. Organizational Behavior \& Human Decision Processes 69:237-249.

Yaniv I, Foster DP. 1997. Precision and accuracy of judgmental estimation. Journal of Behavioral Decision Making 10:21-32. 